\title{
A pre-partitioned adaptive chemistry methodology for the efficient implementation of combustion chemistry in particle PDF methods
}

\author{
Y. Liang*, S. B. Pope, P. Pepiot \\ Sibley School of Mechanical and Aerospace Engineering, Cornell University, NY 14853, USA
}

\begin{abstract}
Large Eddy Simulation/particle Probability Density Function (LES/PDF) approaches are now well developed, and can be applied to turbulent combustion problems involving complex flows with strong turbulence-chemistry interactions. However, these methods are computationally expensive, restricting their use to simple fuels with relatively small detailed chemical mechanisms. To mitigate the cost in both CPU time and storage requirements, an adaptive strategy tailored for particle PDF methods is presented here, which provides for each particle a specialized reduced representation and kinetic model adjusted to its changing composition. Rather than performing chemical reduction at runtime to determine the optimal set of equations to use for a given particle, an analysis of the composition space likely to be accessed during the combustion simulation is performed in a pre-processing stage using simple Partially Stirred Reactor (PaSR) computations. In the pre-processing stage, the composition space is partitioned into a user-specified number of regions, over which suitable reduced chemical representations and kinetic models are generated automatically using the Directed Relation Graph with Error Propagation (DRGEP) reduction technique. A computational particle in the combustion simulation then carries only the variables present in the reduced representation and evolves according to the reduced kinetic model corresponding to the composition space region the particle belongs to. This region is identified efficiently using a low-dimensional binary-tree search algorithm, thereby keeping the run-time overhead associated with the adaptive strategy to a minimum. The performance of the algorithm is characterized for propane/air combustion in a PaSR with pairwise mixing. The results show that the reduction errors are well controlled by the specified error tolerance, and that the adaptive framework provides significant gains in cost and storage compared to traditional non-adaptive reduction approaches.
\end{abstract}

Keywords: Adaptive chemistry, Reduced kinetics, LES/PDF, Partially Stirred Reactor, DRGEP

\footnotetext{
* Corresponding Author

Email: y1956@cornell.edu
} 


\section{Nomenclature}

$\mathbb{C}$

$\mathcal{C}$

C

$C_{A}$

$c_{\mathcal{D}}$

$\mathcal{D}$

D

$\mathbb{F}$

$\mathbb{F}_{J}$

H

$h$

$\mathcal{H}_{\mathcal{D}}$

$\mathcal{J}$

$M_{D}$

$M_{J}$

$\dot{m}_{k}^{\text {str }}$

$n_{C}$

$n_{\mathcal{D}}$

$n_{e}$

$n_{\text {in }}$

$n_{p}$

$n_{\text {pair }}$

$N_{R}$

$n_{r}$

$n_{r}^{J}$

Classifying space, of dimension $n_{C}$

Mapping function between full $\mathbb{F}$ and classifying $\mathbb{C}$ spaces

Classification vector in $\mathbb{C}$, of dimension $n_{C}$

Consumption rate of species $A$

Kinetic inhomogeneity cost of the set $\mathcal{D}$

Database of composition vectors $\boldsymbol{\Phi}$

Distance between two reduced models

Full composition space, of dimension $n_{s}+1$

Reduced composition space for model $M_{J}$, of dimension $n_{s}^{J}+1$

Hyperplane

Specific enthalpy

Kinetic homogeneity of the database $\mathcal{D}$

Classification function

Detailed chemical mechanism

Reduced kinetic model for region $R_{J}$

Normalized mass flow rate of the $k$ th inflowing stream in the PaSR

Number of classifier variables; dimension of $\mathbb{C}$

Number of compositions in the database $\mathcal{D}$

Number of elements in the detailed chemical mechanism

Number of inflowing particles per time step in the PaSR

Number of particles in the PaSR

Number of particles changing partners per time step in the PaSR

Number of regions in the partition of the classifying space

Number of reactions in the detailed mechanism

Number of reactions in the reduced kinetic model $M_{J}$ 


\begin{tabular}{|c|c|}
\hline$n_{s}$ & Number of species in the detailed chemical mechanism \\
\hline$n_{s}^{J}$ & Number of species in the reduced kinetic model $M_{J}$ \\
\hline$n_{t}$ & Number of time steps in the simulation \\
\hline$n_{\mathrm{str}}$ & Number of inflowing streams in the PaSR \\
\hline$n_{T}$ & Number of targets used in DRGEP \\
\hline $\mathbf{P}$ & Cutting hyperplane anchor point \\
\hline$p$ & Pressure \\
\hline$P_{A}$ & Production rate of species $A$ \\
\hline$P_{a}$ & Pseudo-production rate of atom $a$ \\
\hline $\mathbb{R}$ & DRGEP coefficient space, of dimension $n_{s}$ \\
\hline$R_{A B}$ & DRGEP path-dependent coefficient between species $A$ and $B$ \\
\hline$r_{A B}$ & DRGEP direct interaction coefficient between species $A$ and $B$ \\
\hline$r_{A B,\{\mathcal{G}\}}$ & DRGEP direct interaction coefficient given a set of removed species $\mathcal{G}$ \\
\hline$r_{A r_{i}}$ & DRGEP direct interaction coefficient between species $A$ and reaction $r_{i}$ \\
\hline $\mathbf{R}^{\mathcal{D}}$ & DRGEP coefficient vector for all compositions in the database $\mathcal{D}$ \\
\hline$\widetilde{\mathbf{R}}^{\mathcal{D}}$ & Scaled DRGEP coefficient vector for all compositions in the database $\mathcal{D}$ \\
\hline $\mathbf{R}^{\mathcal{D}, r}$ & DRGEP reaction coefficient vector for all compositions in the database $\mathcal{D}$ \\
\hline $\mathcal{R}_{F \rightarrow J}$ & Reduction operator \\
\hline$R_{J}$ & $J$ th region in the classifying space partition and in the composition space \\
\hline $\mathcal{R}_{J \rightarrow F}$ & Reconstruction operator \\
\hline $\mathbf{R}^{\Phi}$ & DRGEP coefficient vector for the single composition $\boldsymbol{\Phi}$ \\
\hline$\widetilde{\mathbf{R}}^{\Phi}$ & Scaled DRGEP coefficient vector for the single composition $\boldsymbol{\Phi}$ \\
\hline S & Chemical source term for the detailed chemical mechanism \\
\hline $\mathbf{S}_{J}$ & Chemical source term for the reduced kinetic model $M_{J}$ \\
\hline $\mathcal{T}$ & Set of targets used in DRGEP \\
\hline$T$ & Temperature $[\mathrm{K}]$ \\
\hline
\end{tabular}




\begin{tabular}{|c|c|}
\hline$t$ & Time $[\mathrm{s}]$ \\
\hline V & Cutting hyperplane normal vector \\
\hline $\mathbf{Y}$ & Full species mass fraction vector, of dimension $n_{s}$ \\
\hline $\mathbf{y}_{J}$ & Mass fraction vector in the representation $\phi_{J}$, of dimension $n_{s}^{J}$ \\
\hline$\widehat{\mathbf{y}}$ & Mass fractions of locally-significant species \\
\hline$\alpha_{T, \Phi}$ & DRGEP scaling coefficient for target $T$ and composition $\mathbf{\Phi}$ \\
\hline$\Delta t$ & Simulation time step $[\mathrm{s}]$ \\
\hline$\varepsilon$ & Reaction-mapping error for a reduced kinetic model \\
\hline$\varepsilon_{c}$ & Specified error tolerance \\
\hline$\varepsilon_{S A}^{A}$ & Sensitivity coefficient of species $A$ in Reduced Modeling \\
\hline$\varepsilon_{\text {sig }}$ & $\begin{array}{l}\text { Threshold for the mass fraction of an inactive species to be regionally or } \\
\text { locally significant }\end{array}$ \\
\hline$\varepsilon_{\Phi}$ & Reaction mapping error for composition $\boldsymbol{\Phi}$ \\
\hline$\varepsilon_{X}$ & Incurred error in $X$ in a PaSR simulation \\
\hline$\hat{\varepsilon}_{X}$ & Conservation error in $X$ in a PaSR simulation \\
\hline $\boldsymbol{\theta}_{J}$ & Set of species in the reduced kinetic model $M_{J}$ \\
\hline$\Theta$ & Set of species in the detailed mechanism \\
\hline$\widehat{\boldsymbol{\theta}}$ & Set of locally-significant species \\
\hline$\nu_{i, A}$ & Stoichiometric coefficient of species $A$ in reaction $i$ \\
\hline$\xi_{J}$ & Set of reactions in the reduced kinetic model $M_{J}$ \\
\hline$\tau_{\operatorname{mix}}$ & Particle mixing time scale in the PaSR $[\mathrm{s}]$ \\
\hline$\tau_{\text {pair }}$ & Particle pairing time scale in the PaSR $[\mathrm{s}]$ \\
\hline$\tau_{\text {res }}$ & Residence time in the PaSR $[\mathrm{s}]$ \\
\hline$\Upsilon$ & List of models available for a region, parameterized by the model error $\varepsilon$ \\
\hline$\Phi$ & Temperature-based representation of a composition in $\mathbb{F}$ space \\
\hline$\Phi^{D}$ & $\begin{array}{l}\text { Temperature-based representation of a composition in } \mathbb{F} \text { space obtained } \\
\text { using } M_{D}\end{array}$ \\
\hline
\end{tabular}




$\begin{array}{ll}\phi_{J} & \text { Reduced representation based on the reduced kinetic model } M_{J} \\ \boldsymbol{\Phi}^{m} & \begin{array}{l}\text { Temperature-based representation of a composition in } \mathbb{F} \text { space after the } \\ \text { mixing fractional step }\end{array} \\ \boldsymbol{\Phi}^{\prime} & \text { Enthalpy-based representation of a composition in } \mathbb{F} \text { space } \\ \boldsymbol{\Phi}_{k}^{\text {str }} & \text { Composition of } k \text { th inflowing stream in the PaSR } \\ \boldsymbol{\Xi} & \text { Set of reactions in the detailed mechanism } \\ \omega_{i} & \text { Reaction rate of reaction } i\end{array}$

\section{Introduction}

The understanding of chemical kinetics for hydrocarbon fuel combustion has exploded over the past two decades, leading to the development of ever growing detailed kinetic schemes for a wide range of molecular species relevant to hydrocarbon combustion [1]. With more accurate rate rules and improved mechanistic considerations, some of the latest published mechanisms are approaching $10^{4}$ species and typically five times as many reactions [2]. However, these advances in chemical kinetics and detailed model development have to be integrated with Computational Fluid Dynamics (CFD) tools to fully realize their potential in terms of improved understanding and optimization of practical combustion devices. The real challenge then is to maximize the level of chemical detail that can be afforded in CFD. Different modeling approaches for turbulent flows impose different upper limits on the number of variables that can be used to describe the chemistry, ranging from around twenty in Direct Numerical Simulations [3], to a few dozens for LES/particle PDF methods [4], to a few hundreds for flamelet-based approaches [5, 6].

Numerous techniques have been developed to drastically reduce the computation burden associated with a detailed description of the chemistry. A non-exhaustive list includes: (i) Automatic elimination of negligible species and reactions to generate skeletal mechanisms [7, 8, 9, 10, 11, 12, 13]; (ii) Dimension reduction techniques, which further decrease the number of species or degrees of freedom that need be considered, including chemical lumping [14, 15], mathematical lumping [16], QSSA [17, 18], RCCE [19], ILDM [20], REDIM [21] and ICEPIC [22]; and (iii) Storage/retrieval algorithms to reduce the computational cost of repetitive kinetics calculations, including ISAT [23, 24] and PRISM [25]. (One component of the current methodology is DRGEP [9], which is in category (i).) Rather than being in competition, these general approaches can be used in combination with a compounding of the benefits. Yet, reaching the level of reduction necessary to handle complex molecules or multi-component mixtures still appears as a major challenge.

One way forward is to observe that, for a given reactive flow configuration, thermodynamic conditions can vary widely in space and time, and in any small range of temperature and compositions, many species have negligible concentrations, and only a few are chemically active. Hence, the chemistry description in that composition space region may require 
many fewer species and reactions than a more detailed model applicable over the entire composition space. This observation leads to the concept of adaptive chemistry, in which the chemical representation and kinetic equations are adapted to the local chemical activity and thermodynamic state.

Several attempts to devise computationally-viable adaptive chemistry strategies have

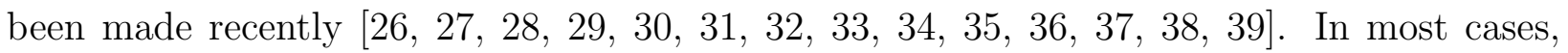
on-the-fly reduction is used, in which at each time step of the simulation, the local composition is analyzed to determine the smallest set of important species to solve for, with the other unimportant species kept as "inert". This methodology theoretically guarantees a maximal reduction in the time needed to integrate the chemistry equations, but is efficient only if the time taken by the reduction algorithm is comparatively small. Species elimination or dimension-reduction methods based on graph or element flux analysis methods have been shown to satisfy this requirements [27, 29, 34, 35, 36, 37, 38, 40]. In particular, the Dynamic Adaptive Chemistry, or DAC, technique [27] has been applied on a variety of configurations, including methane/air combustion in partially-stirred reactor [34], 1D premixed, unsteady, freely-propagating methane/air flame [35], homogeneous ignition [37], and compression-ignition engine simulations [36]. Other on-the-fly reduction approaches considered HCCI engine simulations [39], and even large scale simulations of a turbulent non-premixed ethylene flame [30].

An alternative to on-the-fly reduction is to incorporate some amount of preprocessing calculations to reduce the overhead of the adaptive strategy at runtime. An early demonstration was made by Schwer et al. [26], who manually assembled a small library of reduced models addressing key chemistry regimes expected to be encountered in a flame. Criteria involving local species mass fractions were used to decide which model to use in each domain cell in the simulation of a turbulent hydrogen shear layer flame and an axisymmetric laminar partially premixed methane-air flames. Singer and Green [32] propose a snapshot method to tabulate a priori proper orthogonal decomposition projection matrices, which are then used adaptively at run time to project minor species mass fractions onto a reduced dimensional space, thereby accelerating flame calculations.

While major progress in the adaptive chemistry field has been made in reducing the cost associated with the chemistry calculations, most of the strategies listed above rely on the knowledge of the full composition vector at every given time and location. This avoids the conceptual problems of mechanism augmentation (i.e. re-introducing species) and transport-term evaluation. While storage is not an issue for relatively small kinetic schemes, this approach quickly becomes prohibitively expensive as the number of chemical variables is increased. Tosatto et al. 29] propose a solution to this problem by enabling species removal and re-introduction through a graph analysis modified to account for transport processes. While the integration of the resulting algorithm with flame simulation codes is not straightforward, very good performances are demonstrated in $2 \mathrm{D}$ axisymmetric co-flow flame calculations.

In this paper, we present and demonstrate a pre-partitioned adaptive chemistry strategy to handle large chemical mechanisms and tailored for particle Probability Density Function 
(PDF) methods. These methods are now well developed, and can be applied to turbulent combustion problems involving complex flows with strong turbulence-chemistry interactions [41, 42]. In the particle PDF framework, the gas composition within the solution domain is represented by a large number (up to several millions) of notional particles, each carrying one realization of the fluid thermochemical state. One of the virtues of PDF methods is that reaction is in closed form, allowing for realistic chemistry to be incorporated. But that comes at a significant increase in CPU time compared to other approaches and models (e.g., a factor of 3 or more [43]). As such, because the chemistry in PDF methods is described by a detailed (or reduced) mechanism and is treated in isolation in a fractional step, adaptive strategies appropriate for PDF methods to simulate turbulent combustion involve somewhat different issues than the Eulerian framework typically considered in the literature mentioned above, in which the energy and species transport equations, discretized and solved on a grid, require closures for the reaction term that combine both chemical kinetics and turbulence/chemistry interaction assumptions.

Previous work on this problem includes that of Banerjee and Ierapetritou [33], who use elaborate clustering techniques to create a library of reduced kinetic models, and explicitly determine the highly non-convex regions in composition space over which those models are valid. They also detail how such a model library could be used in an adaptive simulation framework, providing proof-of-concept using the simpler Partially Stirred Reactor (PaSR) configuration. The present work also considers the PaSR as its development and validation framework due to its direct relevance to PDF simulations of turbulent combustion. However we adopt a much simpler conceptual approach, in which the composition space is partitioned into judiciously chosen regions prior to the derivation of reduced kinetic models. Computational particles are also constrained to carry only the minimum number of variables needed to represent their thermochemical state accurately, so that both CPU time and memory requirements associated with particle PDF methods are significantly reduced.

The two primary defining characteristics of the methodology are that the composition space is partitioned in a pre-processing step, and that DRGEP is used to generate the reduced kinetic models. The methodology can therefore be called Pre-Partitioned Adaptive Chemistry using Direction Relation Graphs with Error Propagation, or PPAC-DRGEP.

The remainder of this paper is organized as follows. In Sec. 2, we provide a mathematical description of how chemistry is represented in particles PDF computations, using a Partially Stirred Reactor configuration as the framework. In Sec. 3, an overview of the adaptive chemistry methodology is provided, followed by a more detailed description of the reduction technique employed (Sec. 4) and of each component of the algorithm (Sec. 5). The method is then applied to a propane/air non-premixed PaSR, described in Sec. 6, and its performance is analyzed in Sec. 7. Possible improvements and extensions to the methodology, and its application in LES/PDF simulations, are discussed in Sec. 8, A summary and conclusions are provided in Sec. 9 . 


\section{Particle representation in a partially-stirred reactor (PaSR)}

We consider a reacting mixture of ideal gases, consisting of $n_{s}$ chemical species composed of $n_{e}$ elements. For simplicity of exposition, the mixture is assumed to evolve at a fixed pressure $p$, so that (given $p$ ) the full thermochemical state, or composition, of the mixture is completely characterized by the $n_{s}$-vector of species mass fractions $\mathbf{Y}$ and the mixture temperature $T$. Accordingly, we define the composition to be the $\left(n_{s}+1\right)$-vector: $\mathbf{\Phi} \equiv\{\mathbf{Y}, T\}$. We denote by $\mathbb{F}$ the full composition space, so that any composition vector $\boldsymbol{\Phi}$ is an element of $\mathbb{F}$.

In some situations it is convenient to consider the enthalpy $h$ as the energy variable, in place of $T$. We therefore define the alternative composition vector $\boldsymbol{\Phi}^{\prime} \equiv\{\mathbf{Y}, h\}$. These two representation of the composition contain the same information, and it is straightforward to transform from one to the other.

As mentioned above, a partially-stirred reactor (PaSR) is used as a reference configuration to develop, test, and validate the adaptive methodology. The reactor consists of a constant, even number $n_{p}$ of particles, the $n$th particle having composition $\boldsymbol{\Phi}^{(n)}$. Each particle is assigned a partner, and the particles are ordered so that particles $n$ and $n+1$ are partners, for odd $n$. The PaSR is continuously fed by a specified number $n_{\text {str }}$ of inflow streams, the $k$ th stream having prescribed composition $\boldsymbol{\Phi}_{k}^{\text {str }}$, and normalized mass flow rate $\dot{m}_{k}^{\text {str }}$. Also specified are the residence time, $\tau_{\text {res }}$, a mixing time, $\tau_{\text {mix }}$, and a pairing time, $\tau_{\text {pair }}$. The particular PaSR test case considered is described in Sec. 6, where the values of these quantities are given in Table 1 .

The compositions of the particles evolve in time in small time steps, $\Delta t$, in three fractional steps accounting for: inflow/outflow/pairing; mixing; and reaction. In the inflow/outflow/pairing fractional step, an even number, $n_{\text {in }}$, of particles are selected at random with equal probability and their compositions are replaced by inflow stream compositions. In expectation, that is, when considering its long-run average value, $n_{\text {in }}$ is equal to $n_{p} \Delta t / \tau_{\text {res }}$, and the number from each inflow stream is proportional to its specified mass flow rate. A number, $n_{\text {pair }}$, pairs of particles are then selected for pairing. In expectation, $n_{\text {pair }}$ is equal to $\frac{1}{2} n_{p} \Delta t / \tau_{\text {res }}$. These pairs include those involved in inflow/outflow and others selected at random with uniform probability. These particles are then randomly shuffled to reassign partners.

We denote by $\boldsymbol{\Phi}^{\prime(n)}(t)$ and $\boldsymbol{\Phi}^{\prime(n), m}$ the enthalpy-based composition of the $n$th particle before and after the mixing fractional step. Then, mixing amounts to a small exchange of composition between partners:

$$
\begin{aligned}
\boldsymbol{\Phi}^{\prime(n), m} & =\boldsymbol{\Phi}^{\prime(n)}(t)-\frac{\Delta t}{\tau_{\text {mix }}}\left(\boldsymbol{\Phi}^{\prime(n)}(t)-\boldsymbol{\Phi}^{\prime(n+1)}(t)\right) \\
\boldsymbol{\Phi}^{\prime(n+1), m} & =\boldsymbol{\Phi}^{\prime(n+1)}(t)-\frac{\Delta t}{\tau_{\text {mix }}}\left(\boldsymbol{\Phi}^{\prime(n+1)}(t)-\boldsymbol{\Phi}^{\prime(n)}(t)\right),
\end{aligned}
$$

for all odd $n$.

At the start of the reaction fractional step, the compositions after mixing, $\boldsymbol{\Phi}^{\prime(n), m}$, are converted to their temperature representations $\Phi^{(n), m}$. Starting from these initial conditions, 
the compositions then evolve due to reaction under isobaric, adiabatic conditions over a time $\Delta t$ according to

$$
\frac{d \boldsymbol{\Phi}^{(n)}(t)}{d t}=\mathbf{S}\left(\boldsymbol{\Phi}^{(n)}(t)\right)
$$

where $\mathbf{S}$ is the chemical source term defined by the user-provided detailed chemical mechanism. This mechanism, denoted by $M_{D}$, involves the set of $n_{s}$ species $\boldsymbol{\Theta}$, and a set of $n_{r}$ reactions $\boldsymbol{\Xi}$. The reaction fractional step yields the final particle composition at $t+\Delta t$, i.e., $\boldsymbol{\Phi}^{(n)}(t+\Delta t)$; and the mapping that Eq. 2 provides from $\boldsymbol{\Phi}^{\prime(n), m}$ to $\boldsymbol{\Phi}^{(n)}(t+\Delta t)$ is referred to as the reaction mapping.

Figure 1 shows the evolution of the temperature of a typical particle in the PaSR. Inflow/outflow events result in a discontinuity of the temperature; pairing results in a discontinuity in $d T / d t$; and in between these discrete events, mixing and reaction result in a smooth evolution.

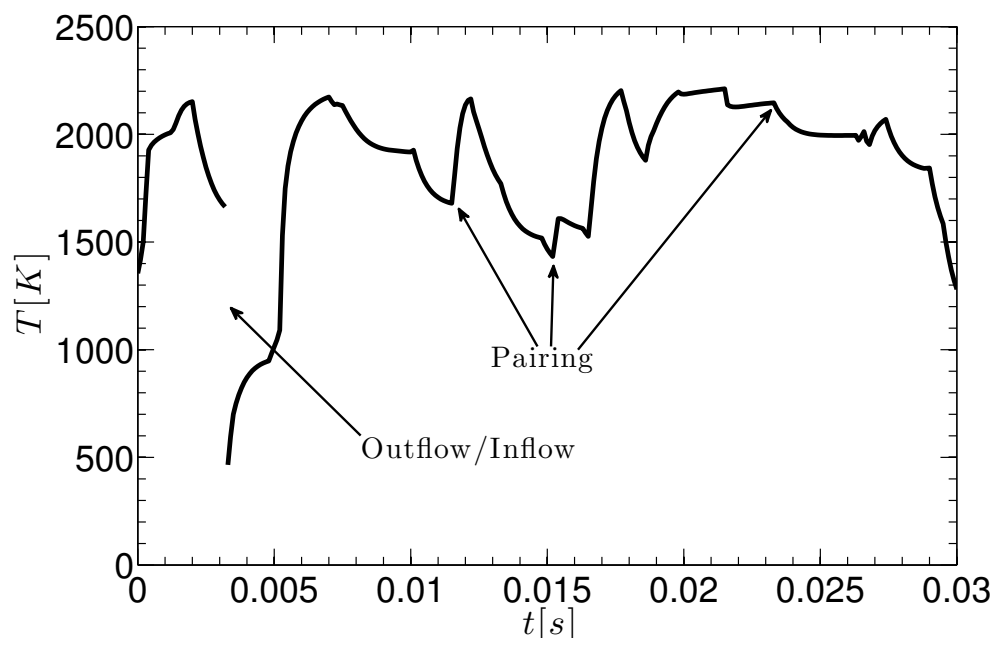

Figure 1: Typical temperature evolution for an individual particle in a PaSR. Discontinuities in $T$ correspond to inflow/outflow events, while discontinuities in $d T / d t$ are associated with pairing events. Apart from those discrete, random events, the temperature evolves continuously due to mixing and reaction. (Only some of the pairing events are indicated.)

\section{Overview of the pre-conditioned adaptive chemistry methodology}

The overall pre-conditioned adaptive chemistry methodology combines an off-line, preprocessing stage (during which the composition space is partitioned and a set reduced kinetic models is created), and an online, dynamic procedure to identify, at time $t$, which reduced model to use for each particle in the combustion simulation. We refer to these two stages as the pre-processing stage and the adaptive simulation. 


\subsection{Pre-processing stage}

The pre-processing stage consists of the following three tasks:

1. Database creation: Using the PaSR and the detailed chemical mechanism, generate a database $\mathcal{D}$ consisting of a large number $n_{\mathcal{D}}$ of full compositions, representative of the compositions that occur during the simulation of interest.

2. Partitioning: Define a low-dimensional space $\mathbb{C}$, referred to hereafter as the classifying space, and a function mapping the composition space $\mathbb{F}$ to the classifying space $\mathbb{C}$, $\mathcal{C}: \mathbb{F} \rightarrow \mathbb{C}$. Partition $\mathbb{C}$ into a specified number $N_{R}$ of regions, the $J$ th region being denoted by $R_{J}$. This also implicitly partitions the composition space $\mathbb{F}$ : corresponding to $R_{J}$, the $J$ th region in $\mathbb{F}$ is all points mapped by $\mathcal{C}$ to $R_{J}$. Henceforth we use $R_{J}$ to denote the $J$ th region in either $\mathbb{C}$ or $\mathbb{F}$, depending on the context.

3. Reduced modeling: For each region $R_{J}$, identify a reduced composition space $\mathbb{F}_{J}$ (a sub-space of $\mathbb{F}$ ), a reduced representation of the composition, $\phi_{J} \equiv\left\{\mathbf{y}_{J}, T\right\} \in \mathbb{F}_{J}$, and an appropriate reduced kinetic model $M_{J}$. The reduced representation and kinetic model involve a reduced set of $n_{s}^{J}$ retained species $\boldsymbol{\theta}_{J}$ and a reduced set of $n_{r}^{J}$ retained reactions $\boldsymbol{\xi}_{J}$, with the expectation that $n_{s}^{J}$ and $n_{r}^{J}$ are significantly smaller that $n_{s}$ and $n_{r}$, respectively. The $n_{s}^{J}$-vector of the mass fraction of the retained species is denoted by $\mathbf{y}_{J}$. This procedure is called reduced modeling mapping for $R_{J}$ :

$$
M_{J} \equiv\left\{\boldsymbol{\phi}_{J}, \boldsymbol{\theta}_{J}, \boldsymbol{\xi}_{J}\right\} .
$$

\subsection{Reduced representation of compositions}

In the adaptive simulation, the reduced composition of the $n$th particle is denoted by $\phi^{(n)}$ and is defined as

$$
\phi^{(n)}=\left\{J^{(n)}, \mathbf{y}_{J}^{(n)}, \widehat{\boldsymbol{\theta}}^{(n)}, \widehat{\mathbf{y}}^{(n)}, T^{(n)}\right\},
$$

where $J$ indicates that the representation is based on model $M_{J}$, and $T$ is the temperature. The way the species are represented in Eq. 4 is schematically presented in Fig. 2, and further explanation is provided below.

As described in Sec. 5.3, the DRGEP procedure used to generate the model $M_{J}$ identifies which species are kinetically active, and the rest are deemed to be inactive. Of the inactive species, some may be deemed to be regionally significant, that is, significant for a given region $R_{J}$ in composition space, if their mass fraction has a significant probability to be above a specified threshold $\varepsilon_{\text {sig }}$ (taken to be $\varepsilon_{\text {sig }}=10^{-3}$ ) among compositions in the region. This is further explained and made precise in Sec.5.3. Both active and regionally-significant species are included in $M_{J}$, and their mass fractions are denoted by $\mathbf{y}_{J}$.

An inactive species that is not regionally significant is deemed to be locally significant, that is, significant for a specific composition, if its mass fraction in this composition exceeds

$\varepsilon_{\text {sig. }}$. In the reduced representation, Eq. $4, \widehat{\boldsymbol{\theta}}$ denotes the set of locally-significant species, and $\hat{\mathbf{y}}$ denotes their mass fractions. It is found to be advantageous in terms of accuracy and efficiency to retain these locally-significant species in the particle composition, but not in the model $M_{J}$ : neglecting these species leads to inaccuracy, while including them in the model leads to unduly large models and hence inefficiency. It is expected (and found in practice) 


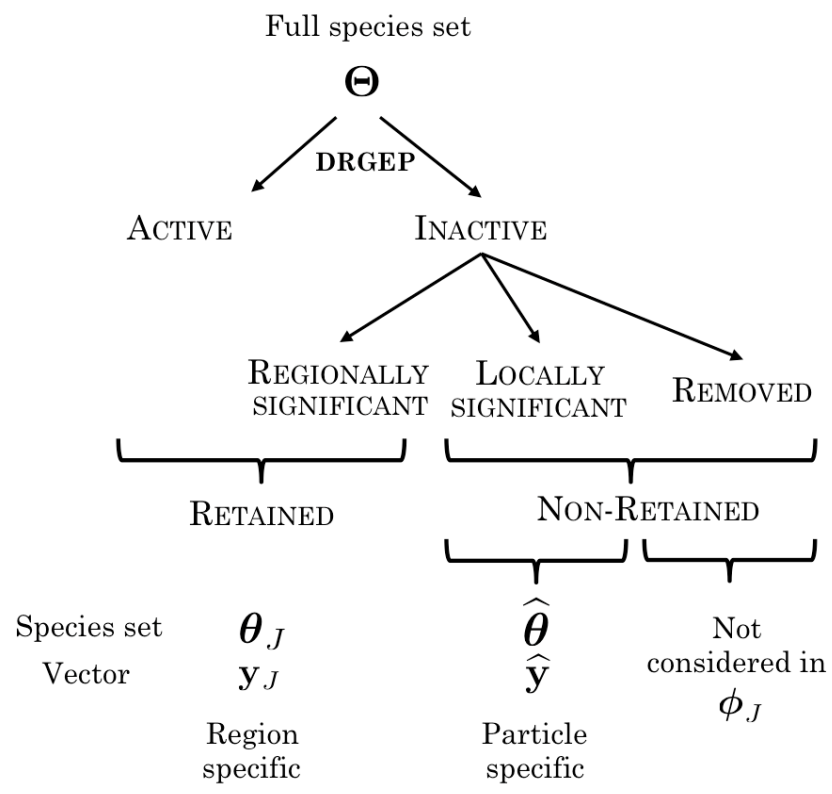

Figure 2: Species nomenclature used in the reduced representation of compositions. Note that $\boldsymbol{\xi}_{J}$, the set of retained reactions for region $J$, involves active species exclusively, with the exception of third-body expressions that may include regionally and locally significant species.

that locally-significant species are seldom needed. It should be noted that to satisfy the normalization condition for mass fractions, the components of $\mathbf{y}_{J}$ and $\hat{\mathbf{y}}$ together sum to unity.

Henceforth, we abbreviate the notation (Eq. 4p for the reduced representation of composition to:

$$
\phi_{J}^{(n)}=\left\{\mathbf{y}_{J}^{(n)}, \widehat{\mathbf{y}}^{(n)}, T^{(n)}\right\}
$$

it being implied that $J^{(n)}$ and $\widehat{\boldsymbol{\theta}}^{(n)}$ are also known. We refer to this as the reduced composition based on model $M_{J}$, and note that it does not necessarily require that the composition belongs to region $R_{J}$.

\subsection{Adaptive simulation}

Once the classifying space partition and a corresponding set of reduced kinetic models $M_{J=1, \ldots, N_{R}}$ have been constructed, the time evolution of a particle composition in the PaSR can be computed using reduced representations and kinetic models dynamically chosen among this set. This adaptive algorithm, illustrated in Fig. 3 for $N_{R}=4$, proceeds in the following sub-steps:

1. At the beginning of each time step, the composition of particle $n$ is expressed using the reduced representation based on model $M_{J}$ (for some known $J$ ): $\phi_{J}^{(n)}(t)=$ $\left\{\mathbf{y}_{J}^{(n)}(t), \widehat{\mathbf{y}}^{(n)}(t), T^{(n)}(t)\right\}$.

2. In the mixing fractional step involving two paired particles $n$ and $n+1$ with reduced compositions $\phi_{J}^{(n)}$ and $\phi_{K}^{(n+1)}$ pertaining to models $M_{J}$ and $M_{K}$, respectively, the full 
representations $\boldsymbol{\Phi}^{(n)}$ and $\boldsymbol{\Phi}^{(n+1)}$ are first reconstructed. These reconstructed compositions are then mixed according to Eq. 1 to yield the full compositions after mixing, denoted by $\boldsymbol{\Phi}^{(n), m}$ and $\boldsymbol{\Phi}^{(n+1), m}$.

3. The classification algorithm is used to identify which regions $R_{J^{\prime}}$ and $R_{K^{\prime}}$ that $\boldsymbol{\Phi}^{(n), m}$ and $\boldsymbol{\Phi}^{(n+1), m}$ belong to.

4. The full compositions $\boldsymbol{\Phi}^{(n), m}$ and $\boldsymbol{\Phi}^{(n+1), m}$ are then reduced to the reduced representations $\boldsymbol{\phi}_{J^{\prime}}^{(n), m}$ and $\boldsymbol{\phi}_{K^{\prime}}^{(n+1), m}$, based on models $M_{J^{\prime}}$ and $M_{K^{\prime}}$.

5. Finally, in the reaction fractional step, the reduced representation of particle $n$ located in region $R_{J}, \phi_{J}^{(n)}$, is integrated over the time increment $\Delta t$ according to the reduced kinetic model $M_{J}$. These values of $J$ and $\phi_{J}^{(n)}$ are then used in sub-step 1 of the next time step. How the locally-significant species are treated in the reaction fractional step is described in Sec. 5.3 .

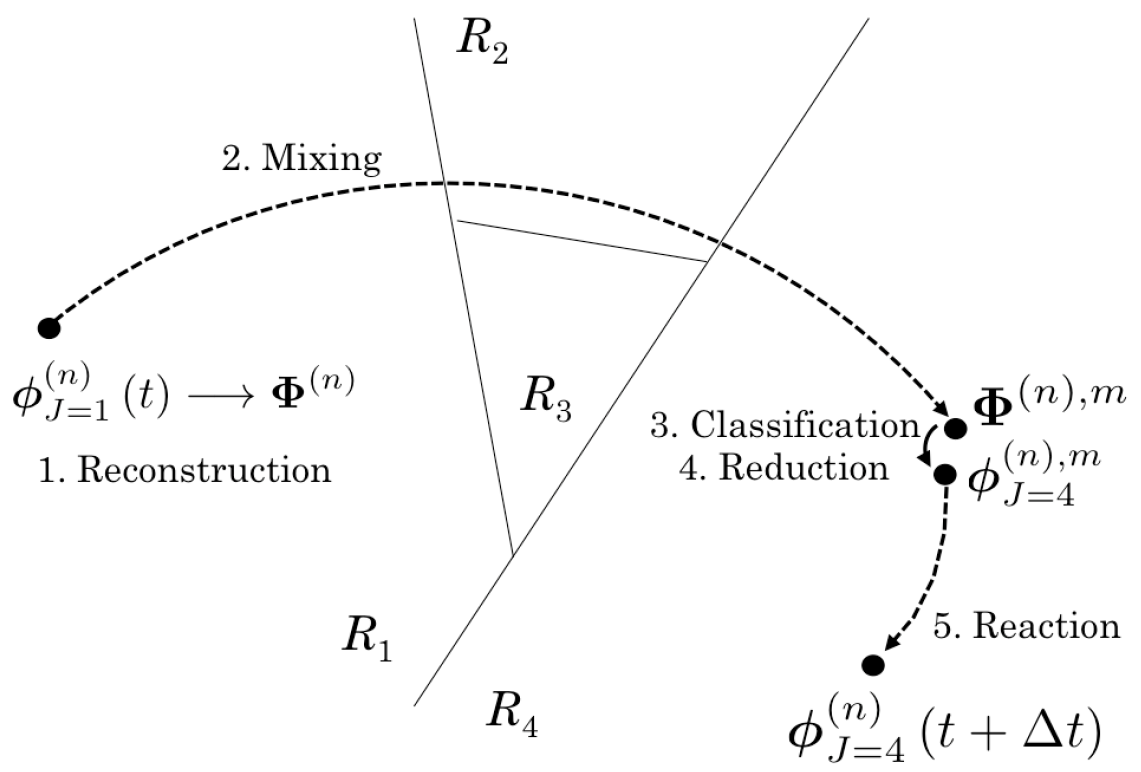

Figure 3: Overview of the adaptive strategy. The classifying space $\mathbb{C}$ is partitioned into $N_{R}=4$ regions, $R_{1}$ to $R_{4}$, for which there are corresponding reduced kinetic models $M_{1}$ to $M_{4}$. At the beginning of the time step, particle $n$, represented by the black circle, has the reduced representation based on model $M_{1}$. In the mixing fractional step, the reduced representation $\phi_{J=1}^{(n)}$ is first reconstructed to a full representation $\boldsymbol{\Phi}^{(n)}$ (1), and mixed using the pairwise mixing model of Eq. 1 (2). Because of the mixing process, the full representation after mixing, $\boldsymbol{\Phi}^{(n), m}$, has moved to region $R_{4}$, as determined by classification (3). $\boldsymbol{\Phi}^{(n), m}$ is reduced to $\phi_{J=4}^{(n), m}(4)$, then integrated in time according to the reduced model $M_{4}(5)$ to yield the particle composition at the end of the time step: $\phi_{J=4}^{(n)}(t+\Delta t)$. (Note that the final composition, although based on model $M_{4}$ is not necessarily in region $R_{4}$ ). 


\subsection{Required operations}

The adaptive procedure outlined above requires the definition of the following generic operations, applicable to any particle with reduced composition $\phi_{J}$. Detailed descriptions and implementations are provided in Section 5 .

1. Reconstruction: The full $(F)$ representation $\Phi \in \mathbb{F}$, reconstructed from the reduced representation $\phi_{J}$, is provided by:

$$
\Phi=\mathcal{R}_{J \rightarrow F}\left(\phi_{J}\right) .
$$

2. Classification: Given a full representation $\boldsymbol{\Phi}$ of an individual particle composition, the classification function $\mathcal{J}$ that returns the index of the region it belongs to is defined as:

$$
J=\mathcal{J}(\Phi) .
$$

3. Reduction: The full representation $\mathbf{\Phi}$ of a given particle composition can be converted to the reduced representation based on model $M_{J}$ by the reduction operation:

$$
\phi_{J}=\mathcal{R}_{F \rightarrow J}(\Phi) .
$$

Note that information is lost in the reduction, so a subsequent reconstruction does not (in general) recover the original composition, i.e., $\boldsymbol{\Phi} \neq \mathcal{R}_{J \rightarrow F}\left(\mathcal{R}_{F \rightarrow J}(\boldsymbol{\Phi})\right)$. This loss of information in the reduction process leads to an error in the adaptive simulation, which is referred to as conversion error. On the other hand, reconstruction is done consistently to satisfy $\phi_{J}=\mathcal{R}_{F \rightarrow J}\left(\mathcal{R}_{J \rightarrow F}\left(\phi_{J}\right)\right)$.

In the following sections, we first provide (in Sec. 4) an overview of the Directed Relation Graph with Error Propagation method, which lies at the core of this adaptive chemistry methodology. We then describe in detail (in Sec. 5) the algorithms required at each stage, namely our approach to:

- In the pre-processing stage: generating a database $\mathcal{D}$ of representative compositions; identifying a suitable classifying space $\mathbb{C}$; subsequently partitioning $\mathbb{C}$ into $N_{R}$ regions (Partitioning); and, developing accurate reduced kinetic models for each of these regions (Reduced Modeling);

- In the adaptive simulation stage: Reconstructing the full representation of a given particle from its reduced representation (Reconstruction), determining the region a particle belongs to based on its current representation (Classification), and finally converting a particle composition from a full reconstructed representation to its appropriate reduced representation (Reduction).

The reader less interested in the details of the implementation may want to skip to Sec. 6 . 


\section{Directed relation graph with error propagation (DRGEP)}

By analyzing production rates derived from an ensemble of composition states, the Directed Relation Graph with Error Propagation (DRGEP) method [9] quantifies the coupling between the species and reactions included in a chemical mechanism and some user-specified targets. The main output from this analysis is a set of importance coefficients, which are referred to as DRGEP coefficients, allowing us to rank species and reactions from most important to least important for the prediction of the chosen targets. DRGEP coefficients are used at two different levels in the pre-processing stage: i) in the Partitioning stage, to identify regions in composition space that share common chemical characteristics; and ii) in the Reduced Modeling stage, to derive region-specific reduced kinetic models. The main ingredients of the DRGEP method are recalled below, the first one being the determination of a set of $n_{T}$ targets $\mathcal{T}$, most often particular species or heat release, on which we want to quantify the influence of individual species and reactions.

Direct interaction coefficients. Direct interaction coefficients are defined as the measure of the coupling between two species that are directly related through an elementary reaction, that is, two species that appear concurrently in the same reaction. For a given composition $\boldsymbol{\Phi}$ and kinetic model $M$, the coupling coefficient between two such species $A$ and $B$ is expressed as:

$$
r_{A B} \equiv \frac{\left|\sum_{i=1, n_{R}} \nu_{i, A} \omega_{i} \delta_{B}^{i}\right|}{\max \left(P_{A}, C_{A}\right)},
$$

where the production and consumption of species $A$ are defined as:

$$
\begin{aligned}
P_{A} & =\sum_{i=1, n_{R}} \max \left(0, \nu_{i, A} \omega_{i}\right), \\
C_{A} & =\sum_{i=1, n_{R}} \max \left(0,-\nu_{i, A} \omega_{i}\right) .
\end{aligned}
$$

In the above equations, $\omega_{i}$ are the net reaction rate of the $i^{\text {th }}$ reaction evaluated from composition $\boldsymbol{\Phi}$ and kinetic model $M, \nu_{i, A}$ is the stoichiometric coefficient of species $A$ in reaction $i$, and

$$
\delta_{B}^{i}= \begin{cases}1 & \text { if the } i^{\text {th }} \text { reaction involves species } B \\ 0 & \text { otherwise }\end{cases}
$$

Equation 9 can be extended to better quantify the impact of removing a species in addition to a previously removed set of species [9]:

$$
r_{A B,\{\mathcal{G}\}} \equiv \frac{\left|\sum_{i=1, n_{R}} \nu_{i, A} \omega_{i} \delta_{B,\{\mathcal{G}\}}^{i}\right|}{\max \left(P_{A}, C_{A}\right)},
$$

where $\{\mathcal{G}\}$ is the set of species already removed. $\delta_{B,\{\mathcal{G}\}}^{i}$ is unity, if the $i^{\text {th }}$ reaction involves $B$ or any species in subset $\{\mathcal{G}\}$, and is zero otherwise. 
Path-dependent coefficients. To go beyond direct interactions, DRGEP defines path-dependent coefficients that quantify the coupling between any directly or indirectly related species $A$ and $B$. Assuming geometric damping, and again, for a given composition $\boldsymbol{\Phi}$ and kinetic model $M$, the coupling between $A$ and $B$ through a reaction path $p$ is written as:

$$
r_{A B, p}=\prod_{i=1}^{n-1} r_{S_{i} S_{i+1}}
$$

with $S_{1}=A, S_{n}=B, S_{i}$ is on the reaction path $p$ that links $A$ and $B, n$ is the number of reactions involved in path $p$. Since many paths can exist linking $A$ to $B$, only the most important one is retained:

$$
R_{A B} \equiv \max _{\text {all paths } p} r_{A B, p}
$$

$R_{A B}$ is calculated following the implementation used in [9]. Briefly, starting from target $A$, all possible paths of degree $n, n$ being the number of edges separating $A$ from a given species $B$ are explored successively, starting at $n=1$, and $R_{A B}$ tracks the maximum $r_{A B, p}$ encountered on those paths. The algorithm stops when paths of degree $n+1$ return coefficients below a very small cut-off number, guaranteeing that none of the higher-degree paths would be yield $r_{A B, p}$ values larger than the current maximum. Additional details on graph search algorithms in this specific context can be found in [44]. $R_{A B}$ can be interpreted as the magnitude of the error made in the prediction of species $A$ if species $B$ is removed [9].

Target-specific coefficients. In a similar way, the overall importance of $B$ to the target set $\mathcal{T}$ for a given composition $\boldsymbol{\Phi}$ and kinetic model $M$ is defined by:

$$
R_{B, \Phi} \equiv \max _{T \in \mathcal{T}} R_{T B}
$$

Extension to multiple composition states. The above results can be extended to an ensemble of compositions $\mathcal{D}$ to yield the DRGEP coefficient of species $B$ over $\mathcal{D}$ :

$$
R_{B}^{\mathcal{D}}=\max _{\mathbf{\Phi} \in \mathcal{D}} R_{B, \Phi}
$$

Whether referring to a single composition $\boldsymbol{\Phi}$ or a set of compositions $\mathcal{D}$, the DRGEP coefficients can be viewed as an $n_{s}$-vector, $\mathbf{R}^{\Phi}$ or $\mathbf{R}^{\mathcal{D}}$, respectively, in an $n_{s}$-dimensional real space, which we term the DRGEP coefficient space and denote by $\mathbb{R}$.

Scaling factors. By construction, the DRGEP coefficients are for a large part independent of the mass fractions of target species present in the compositions used to derive them. An alternative definition with scaling factors is therefore introduced in [9] to account for the relative importance of individual targets among the compositions contained in $\mathcal{D}$. Defined for any composition $\boldsymbol{\Phi}$ in $\mathcal{D}$, these scaling coefficients $\alpha_{T, \Phi}$ are written:

$$
\alpha_{T, \boldsymbol{\Phi}}=\max _{\text {all atoms } a}\left(\frac{\alpha_{T, \boldsymbol{\Phi}}^{a}}{\max _{\boldsymbol{\Phi} \in \mathcal{D}} \alpha_{T, \boldsymbol{\Phi}}^{a}}\right),
$$


where

$$
\alpha_{T, \Phi}^{a}=\frac{N_{a, T}\left|P_{T}-C_{T}\right|}{P_{a}} .
$$

In the above equations, $a$ refers to each element present in the system $(\mathrm{C}, \mathrm{H}, \mathrm{O}$ and $\mathrm{N}$ for hydrocarbon combustion), $N_{a, T}$ is the number of atoms $a$ in target species $T$, and $P_{T}$ and $C_{T}$ are the production and consumption rates, respectively, of any target species $T$ (Eqs. 10 and 11). $P_{a}$ is the pseudo-production rate of atom $a$, defined as

$$
P_{a}=\sum_{\text {all species } A} N_{a, A} \max \left(0, P_{A}-C_{A}\right)
$$

This scaling coefficient is unity for compositions in which the target contributes to its maximum (over all compositions in $\mathcal{D}$ ) to the exchange of atoms between species, and zero when, for instance, the target species has been consumed entirely. Equation 16 is re-defined to include the scaling factor:

$$
\widetilde{R}_{B, \Phi} \equiv \max _{T \in \mathcal{T}} \alpha_{T, \Phi} R_{T B},
$$

the scaled DRGEP coefficients still being defined as in Eq. 17.

$$
\widetilde{R}_{B}^{\mathcal{D}}=\max _{\boldsymbol{\Phi} \in \mathcal{D}} \widetilde{R}_{B, \Phi}
$$

The scaled coefficients vector $\widetilde{\mathbf{R}}^{\mathcal{D}}$ are used in the following, and the tilde is dropped.

DRGEP reaction coefficients. Simply replacing Eq. 9 by

$$
r_{A r_{i}} \equiv \frac{\left|\nu_{i, A} \omega_{i}\right|}{\max \left(P_{A}, C_{A}\right)}
$$

in the above procedure quantifies the importance of individual reactions on the prediction of the targets, resulting in a $n_{r}$-vector $\mathbf{R}^{\mathcal{D}, r}$, used during the reduced modeling stage.

\section{Implementation details}

In the following, we assume that a database $\mathcal{D}$ has been assembled, which contains a large number of full compositions $\boldsymbol{\Phi}$ that densely sample the portion of the composition space relevant for the combustion simulation of interest. The number of compositions in this database is $n_{\mathcal{D}}$. Details on how to obtain such a database using a suitably chosen PaSR are provided in Sec. 6.

\subsection{Classifying space $\mathbb{C}$}

We define the classifying space $\mathbb{C}$, of specified dimension $n_{C}$, by a subset $\mathcal{I}=\left\{I_{1} . . I_{n_{C}}\right\}$ of the composition variables included in $\boldsymbol{\Phi}$ (species in $\Theta$ and temperature), and the injective mapping function $\mathcal{C}$ :

$$
\mathcal{C}: \mathbb{F} \rightarrow \mathbb{C}, \quad \mathcal{C}(\boldsymbol{\Phi})=\mathbf{C}
$$


where the $i$ th component of $\mathbf{C}$ is defined by

$$
C_{i}=\frac{\Phi_{I_{i}}-\Phi_{I_{i}, \min }^{\mathcal{D}}}{\Phi_{I_{i}, \max }^{\mathcal{D}}-\Phi_{I_{i}, \min }^{\mathcal{D}}}
$$

In Eq. 25. $\Phi_{I_{i} \text {, min/max }}^{\mathcal{D}}$ refers to the minimum/maximum values of $\Phi_{I_{i}}$ observed across the entire database $\mathcal{D}$. The subset $\mathcal{I}$ is chosen so that the corresponding variables span the $n_{C^{-}}$ dimensional subspace of $\mathbb{F}$ closest to the $n_{C}$-dimensional principal component space formed by the first $n_{C}$ principal components of the compositions in the database $\mathcal{D}$ with each variable rescaled between 0 and 1 following Eq. 25. More information on this procedure can be found in Yang et al. [45]. This definition of $\mathcal{C}$ satisfies two important constraints: i) $\mathbf{C}$ is cheap to evaluate, which is essential for a computationally-efficient classification method, and ii), the function $\mathcal{C}$ retains most of the compositional variance found in $\mathcal{D}$, which we expect to translate into good selectivity when classifying compositions. In addition, each component of $\mathbf{C}$ is bounded between zero and one.

\subsection{Partitioning}

The objective of the Partitioning algorithm is to partition the classifying space $\mathbb{C}$ into $N_{R}$ regions $R_{J=1, \ldots, N_{R}}$, determined so that for each index $J$, compositions $\boldsymbol{\Phi} \in \mathbb{F}$ that satisfy

$$
\mathcal{J}(\boldsymbol{\Phi})=J
$$

share similar chemical kinetic characteristics. This has two practical implications: i) the compositions in $R_{J}$ can be represented using a much smaller representation $\phi_{J}$; and ii) the short-term time evolution (over time interval $\Delta t$ ) of each of these compositions in $R_{J}$ can be described accurately by a common specialized kinetic model $M_{J}$ much smaller than the original detailed model $M_{D}$. Instead of considering all compositions $\boldsymbol{\Phi} \in \mathbb{F}$ satisfying Eq. 26 to delineate the regions, we first restrict the analysis on the $n_{\mathcal{D}}$ discrete sample compositions contained in the database $\mathcal{D}$ mentioned above. The Partitioning procedure is described in three stages: the first one presents the overall, iterative structure of the algorithm, the second provides the steps required at each iteration, and the third describes how the optimal set of $N_{R}$ regions is chosen.

- Algorithm structure. Cutting hyperplanes $H$ (by definition, of dimension $n_{C}-1$ ) are iteratively introduced in $\mathbb{C}$ to isolate groups of sample compositions with similar shortterm kinetics. The partition structure is recorded in a binary tree, whose nodes contain the hyperplanes equations (fully determined by their normal vector $\mathbf{V}$ and one anchor point $\mathbf{P}$, both $n_{C}$-vectors) and whose leaves are associated with increasingly refined regions and the subset of sample compositions they contain. A two-dimensional illustration of the iterative procedure used to create and store the list of hyperplanes that define each region in $\mathbb{C}$ is provided in Fig. 4 for a database $\mathcal{D}$ containing 6 compositions.

- Iteration step. The same task has to be accomplished during each iteration of the partitioning procedure: given an initial region that contains a subset $\mathcal{D}_{m} \subset \mathcal{D}$ of 


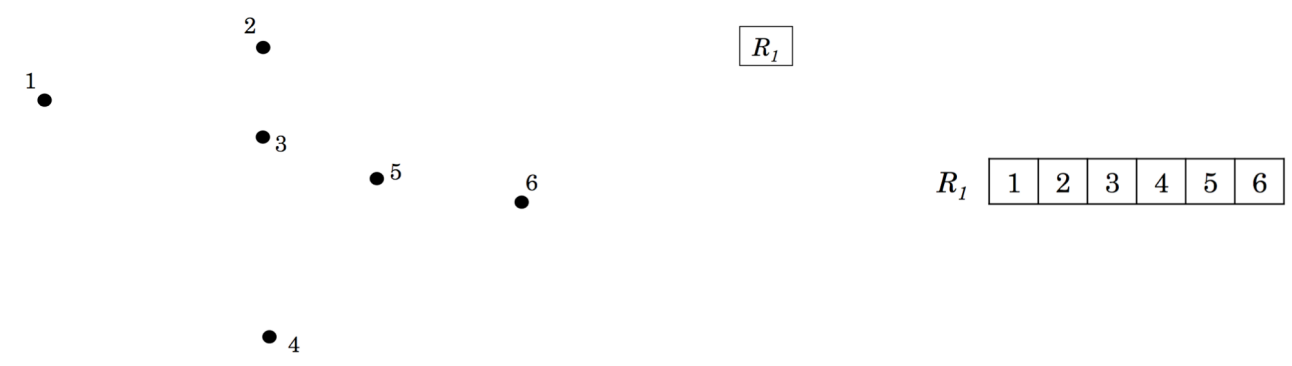

(a) Initial state. Numbered dots indicate the location in $\mathbb{C}$ space of each composition in the database, obtained through $\mathbf{C}^{(n)}=\mathcal{C}\left(\Phi^{(n)}\right), n=1, \ldots, 6$. All compositions initially belong to a single region $R_{1}$.

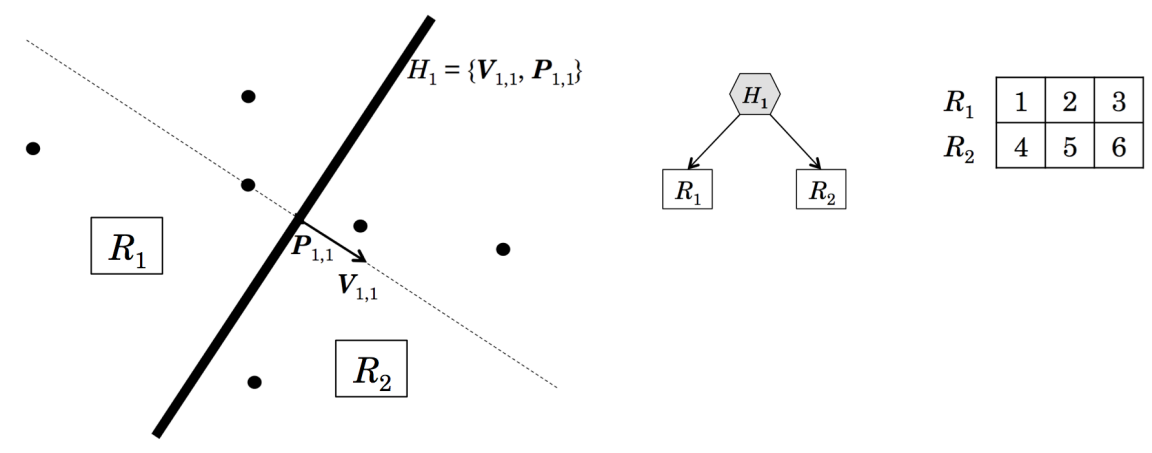

(b) First iteration. A hyperplane $H_{1}$, defined by its normal vector $\boldsymbol{V}_{1,1}$ and anchor point $\mathbf{P}_{1,1}$, is placed in $\mathbb{C}$, creating two distinct regions $R_{1}$ and $R_{2}$. (Note that in each iteration the regions' indices may be re-defined.) The binary tree recording the partition structure now counts one node, containing $H_{1}$, and two leaves, corresponding to $R_{1}$ and $R_{2}$. All points $\mathbf{P}$ in $R_{1}$ satisfy $\left(\mathbf{P}-\mathbf{P}_{1,1}\right) \cdot \boldsymbol{V}_{1,1}<0$, while all points in $R_{2}$ satisfy $\left(\mathbf{P}-\mathbf{P}_{1,1}\right) \cdot \boldsymbol{V}_{1,1} \geqslant 0$. In this example, compositions are split into $\{1,2,3\}$ in $R_{1}$, and $\{4,5,6\}$ in $R_{2}$.

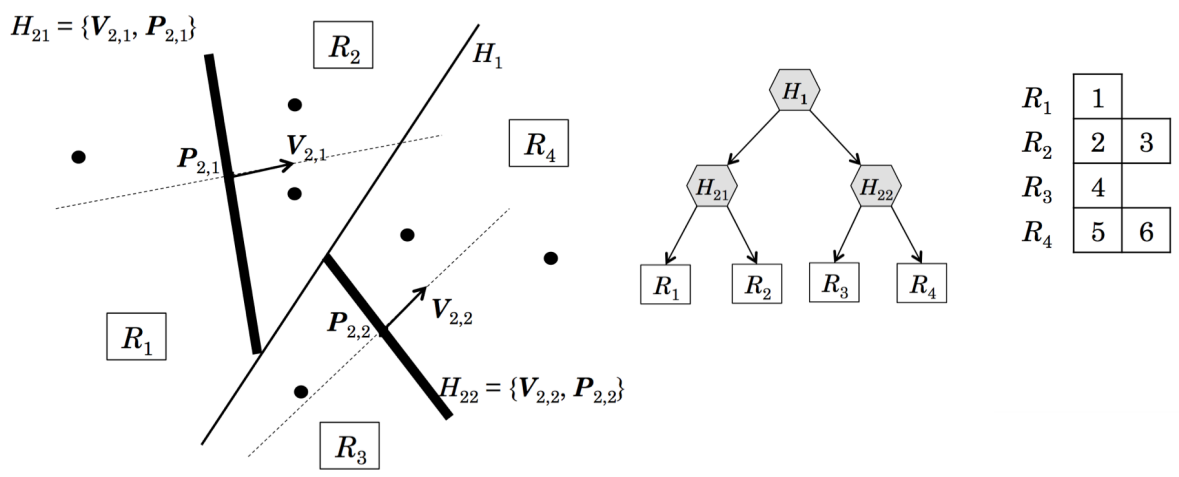

(c) Second iteration. Each previously determined region containing two or more compositions is split further into two subregions using two additional cutting hyperplanes, $H_{21}$ and $H_{22}$. A total of four regions are obtained. The partition binary tree is updated correspondingly. 


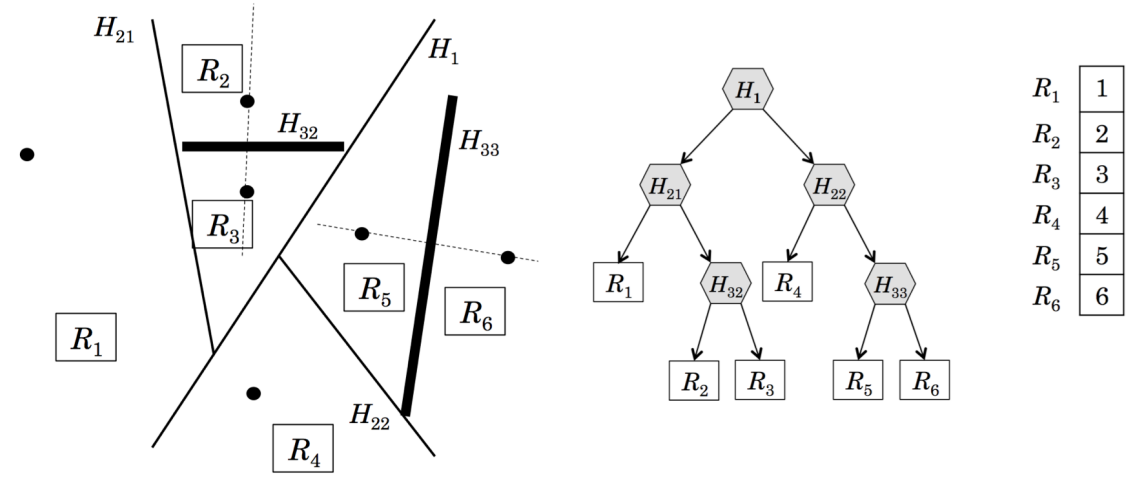

(d) Final iteration. Each region still containing more than one composition is split further. Once each region contains just a single composition, no further refinement is possible. The final result is a binary tree with $n_{\mathcal{D}}$ terminal leaves and $n_{\mathcal{D}}-1$ hyperplane nodes. Compositions belonging to regions far from one another in the tree are expected to have different kinetic characteristics.

Figure 4: Illustration of the iterative partitioning algorithm applied to a two-dimensional classifying space $\mathbb{C}$ and a sample composition database containing 6 elements.

$n_{\mathcal{D}_{m}}$ compositions (determined by a previous iteration, or initially $\mathcal{D}_{m}=\mathcal{D}$ ), find the hyperplane $H$ in $\mathbb{C}$-space that optimally divides the region, and therefore the set of compositions $\mathcal{D}_{m}$, into two sub-regions/subsets with more homogeneous kinetic characteristics. The concept of kinetic homogeneity of a region is defined here as the potential to derive a specialized kinetic model of minimal size able to describe accurately the short-term time evolution of any composition belonging to this region.

We use the positive DRGEP coefficients $\mathbf{R}^{\mathcal{D}_{m}}$ (Eq. 22) defined based on $\mathcal{D}_{m}$ to form an estimate of the kinetic homogeneity of the ensemble of compositions $\mathcal{D}_{m}$ as:

$$
\mathcal{H}_{\mathcal{D}_{m}}=\left\|\mathbf{R}^{\mathcal{D}_{m}}\right\|_{1}=\sum_{i=1}^{n_{s}}\left|R_{i}^{\mathcal{D}_{m}}\right|=\sum_{i=1}^{n_{s}} R_{i}^{\mathcal{D}_{m}} .
$$

The smaller that $\mathcal{H}$ is, the more specialized and reduced the corresponding kinetic model can be, as is briefly justified below. By construction of the DRGEP coefficients, and especially Eq. 22, this definition satisfies the following observations: i) the largest value of $\mathcal{H}$ is obtained for the full composition database $\mathcal{D}$ (no sub-division); ii) the value of $\mathcal{H}$ of any subset of $\mathcal{D}_{m}$ is necessarily equal to or smaller than $\mathcal{H}_{\mathcal{D}_{m}}$; and iii) if all compositions in a set are identical, the homogeneity of any subset is equal to that of the set itself.

Reduced kinetic models tailored for a given set of sample compositions are obtained by removing from the mechanism all species with a DRGEP coefficient less than a userdefined threshold. This threshold can be interpreted as an approximation of the actual prediction error introduced by the reduction [9]. As a consequence, a low value of $\mathcal{H}$ for a set is associated with low DRGEP coefficients and more species removed from the model for a given reduction error threshold. The objective of each iteration is therefore 
to identify a hyperplane $H_{m}$ that divides $\mathcal{D}_{m}$ into two complementary (left and right) subsets, $\mathcal{D}_{m, L}$ and $\mathcal{D}_{m, R}$, contained in subregions $R_{m, L}$ and $R_{m, R}$, respectively, that minimizes the kinetic inhomogeneity, or "cost":

$$
c_{\mathcal{D}_{m}}=\mathcal{H}_{\mathcal{D}_{m, L}}+\mathcal{H}_{\mathcal{D}_{m, R}}=\left\|\mathbf{R}^{\mathcal{D}_{m, L}}\right\|_{1}+\left\|\mathbf{R}^{\mathcal{D}_{m, R}}\right\|_{1}
$$

A global minimum, if it exists, would be computationally prohibitive to obtain given the large number of compositions typically included in the initial database $\mathcal{D}$. Therefore, a simplified approach is adopted here that achieves a significant decrease in the value of $\mathcal{H}$ of each subregion, while remaining computationally affordable. The algorithm is described in Fig. 5 .

- Consolidation into a partition with $N_{R}$ regions. Without any constraints on the number of regions, the iterative partitioning proceeds until $\mathbb{C}$ is partitioned into $n_{\mathcal{D}}$ regions, each of them containing a single composition $\boldsymbol{\Phi}$ from $\mathcal{D}$. To restrict the number of regions to the specified value $N_{R}$, the complete tree is analyzed to identify the upper tree with $N_{R}$ leaves that minimizes

$$
c_{\text {tree }}=\sum_{J=1}^{N_{R}} \mathcal{H}_{\mathcal{D}_{J}},
$$

where the databases $\mathcal{D}_{J}$ are those formed by all compositions $\boldsymbol{\Phi}$ contained in region $J$. This is done in two steps, efficiently implemented using dynamic programming and recursion: in a first pass, the values of $\mathcal{H}$ associated with each leaf and node in the complete tree are computed. By construction, leaves of the complete tree represent regions containing a single composition (denoted by $\boldsymbol{\Phi}_{\text {leaf }}$ ), hence

$$
\mathcal{H}_{\text {leaf }}=\left\|\mathbf{R}^{\boldsymbol{\Phi}_{\text {leaf }}}\right\|_{1}
$$

On the other hand, the value of $\mathcal{H}$ of a node is defined as

$$
\mathcal{H}_{\text {node }}=\left\|\mathbf{R}^{\mathcal{D}_{\text {node }}}\right\|_{1},
$$

where $\mathcal{D}_{\text {node }}$ is the set of compositions in the leaves originating from this node. Once the optimal upper tree is identified, all hyperplanes below the terminal elements of this upper tree are removed, effectively consolidating regions into the specified number $N_{R}$. Figure 6 illustrates the consolidation process on the same partition problem as before, bringing the number of regions from a maximum of 6 to the specified value of $N_{R}=4$.

The final result of this partitioning is a binary tree with a hyperplane $H=\{\mathbf{V}, \mathbf{P}\}$ at each node, and the index $J$ of a distinct region at each leaf. Given a value of the classifying variable $\mathbf{C}$, the tree can be traversed to determine the corresponding region $J$. The tree is traversed by moving to a node's left or right child, depending on the sign of $(\mathbf{C}-\mathbf{P}) \cdot \mathbf{V}$. 
$\mathbb{F}$ space

Composition database $\mathcal{D}_{m}$

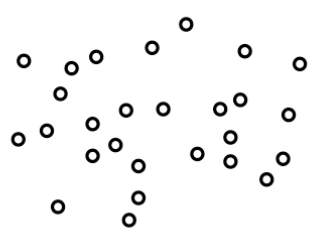

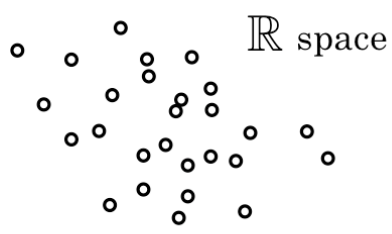

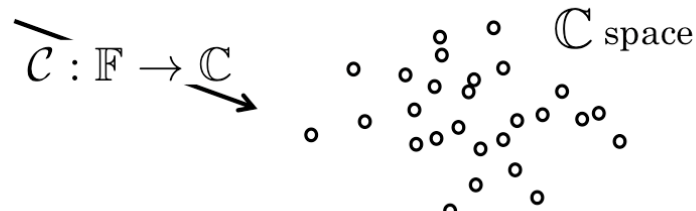

DRGEP $: \mathbb{F} \rightarrow \mathbb{R}$

(a) For each composition $\boldsymbol{\Phi}$ in database $\mathcal{D}_{m}$, the DRGEP coefficients $\mathbf{R}^{\boldsymbol{\Phi}}$ (R space) and the classifying composition $\mathbf{C}(\mathbb{C}$ space) are computed.

$\mathbb{R}$ space

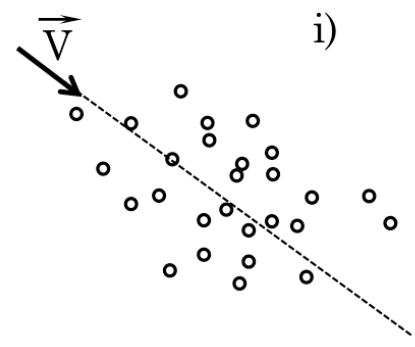

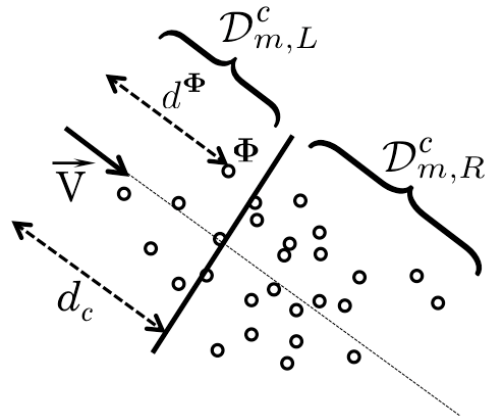

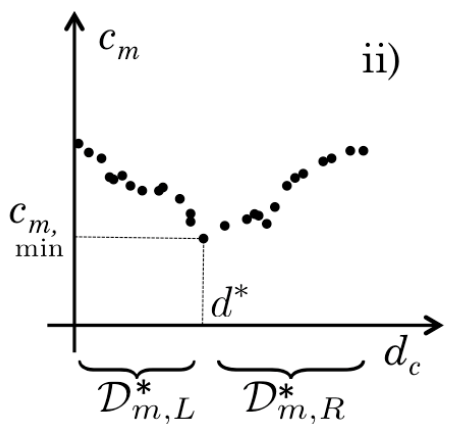

(b) In $\mathbb{R}$ space, the first principal component vector $\mathbf{V}$ of the ensemble of normalized DRGEP coefficients $\widehat{\mathbf{R}}^{\boldsymbol{\Phi}}$, defined as ${\widehat{R_{i}}}^{\boldsymbol{\Phi}}=R_{i}^{\boldsymbol{\Phi}} / \frac{1}{n_{\mathcal{D}}} \sum_{\boldsymbol{\Phi} \in \mathcal{D}_{m}} R_{i}^{\boldsymbol{\Phi}}$ for $i=1, \ldots, n_{S}$, is computed (left). Compositions in $\mathcal{D}_{m}$ are then projected orthogonally onto $\mathbf{V}$ and ranked according to their distance $d^{\mathbf{\Phi}}$ along the direction of $\mathbf{V}$. Sub-databases $\mathcal{D}_{m, L}^{c}$ and $\mathcal{D}_{m, R}^{c}$ are defined for any value $d_{c}$, by separating compositions with $d^{\Phi}<d_{c}$ from those with $d^{\Phi} \geqslant d_{c}$ (middle). The value of $d_{c}, d^{*}$, leading to minimum of $c_{m}$ determines $\mathcal{D}_{m, L}^{*}$ and $\mathcal{D}_{m, R}^{*}$ used in the final step (right).

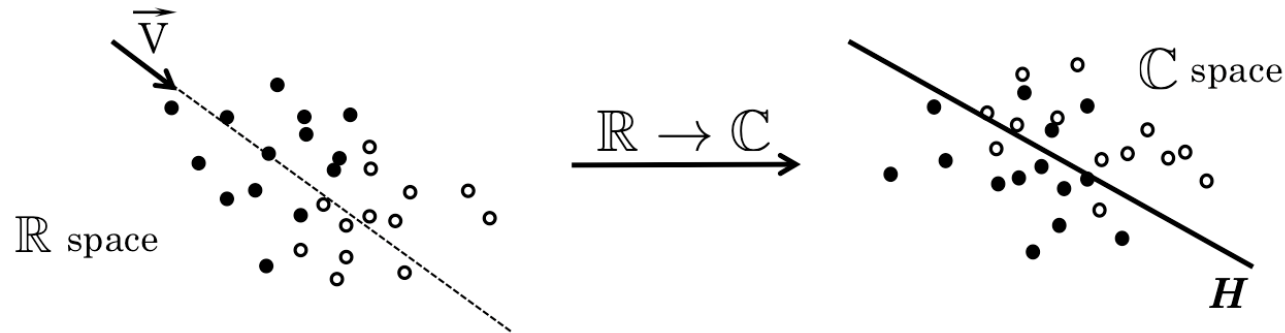

(c) Each composition is colored based on its belonging to $\mathcal{D}_{m, L}^{*}$ or $\mathcal{D}_{m, R}^{*}$. The final step aims at identifying the hyperplane in $\mathbb{C}$-space that best reproduces this coloring, that is, the hyperplane that creates two subdatabases $\mathcal{D}_{m, L}$ and $\mathcal{D}_{m, R}$ as close as possible to $\mathcal{D}_{m, L}^{*}$ and $\mathcal{D}_{m, R}^{*}$. (Note that one-to-one correspondence cannot be achieved in general due to the non-linearity of the relationship between $\boldsymbol{\Phi}$ and $\mathbf{R}^{\boldsymbol{\Phi}}$.) This is done using covariance ellipsoids, as described in Appendix.

Figure 5: Tasks performed at each step of the Partitioning iterative procedure. 


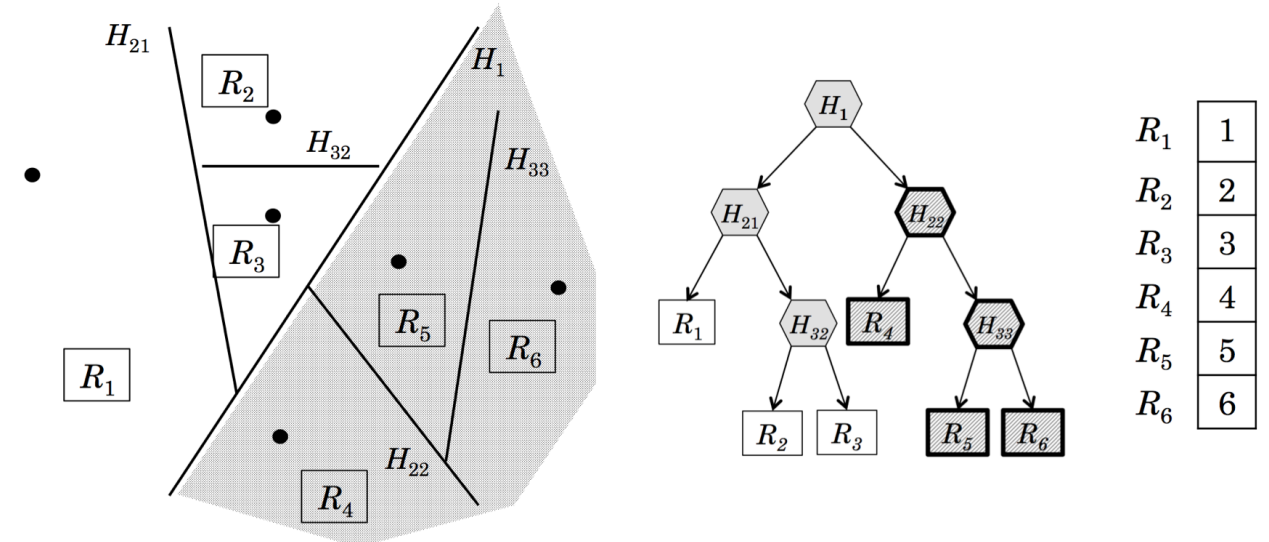

(a) The consolidation procedure first computes the $\mathcal{H}$ values of each element in the tree: the 6 terminal leaves $R_{1}$ to $R_{6}$, and the 5 nodes $H_{1}, H_{21}, H_{22}, H_{32}$, and $H_{33}$. The optimal upper tree is chosen among the 3 possible 4-leaf upper trees of this structure, as that minimizing $c_{\text {tree }}$ (Eq. 29). In this case, $c_{\text {tree }}^{\text {min }}=$ $\mathcal{H}_{\left\{\Phi_{1}\right\}}+\mathcal{H}_{\left\{\boldsymbol{\Phi}_{2}\right\}}+\mathcal{H}_{\left\{\boldsymbol{\Phi}_{3}\right\}}+\mathcal{H}_{\left\{\boldsymbol{\Phi}_{4}, \boldsymbol{\Phi}_{5}, \boldsymbol{\Phi}_{6}\right\}} \cdot$
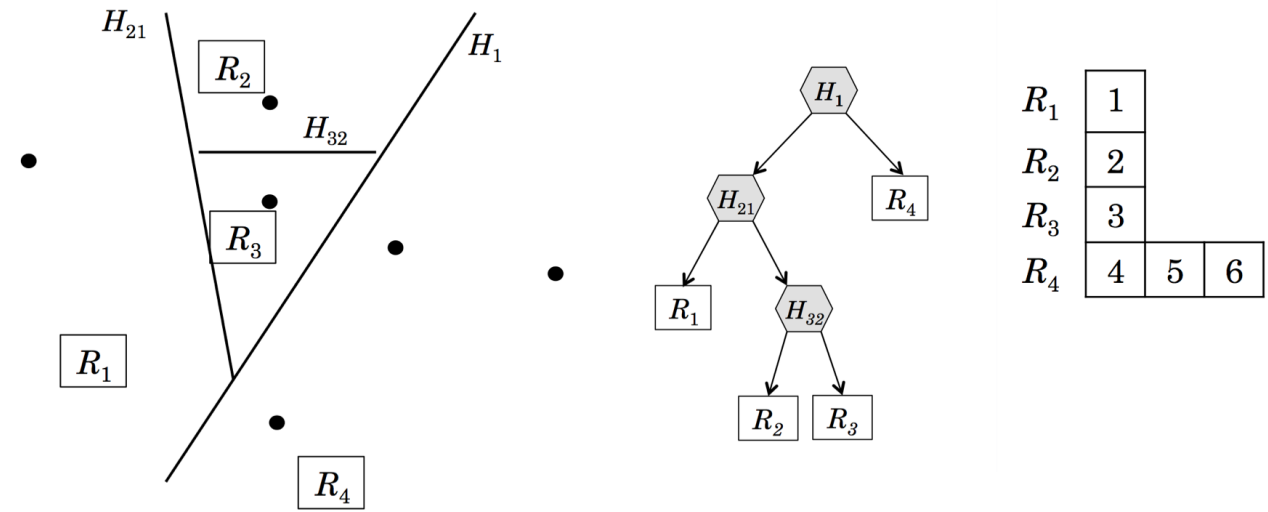

(b) Accordingly, the hyperplane $H_{22}$ and $H_{33}$ are removed, thereby merging regions $R_{4}, R_{5}$, and $R_{6}$. The final Partitioning binary tree is trimmed to the optimal 4-leaf upper tree identified above. Sample compositions are now labeled as belonging to one, and only one, region out of the 4 contained in the partition.

Figure 6: Consolidation of the complete tree obtained in Fig. 4 into an optimal upper tree with $N_{R}=4$ regions.

\subsection{Reduced Modeling}

The Partitioning step establishes the structure of a partition of $\mathbb{C}$ and $\mathbb{F}$ into $N_{R}$ regions, each region $R_{J}$ containing a subset $\mathcal{D}_{J}$ of size $n_{\mathcal{D}_{J}}$ of the original composition database $\mathcal{D}$. The objective of the Reduced Modeling step is to identify a reduced kinetic model $M_{J}$ able to accurately describe the short-term time evolution of any composition belonging to region $R_{J}$. We assume that $n_{\mathcal{D}_{J}}$ is large enough to characterize the chemistry relevant to region $R_{J}$, and therefore it is sufficient to obtain a model $M_{J}$ which is accurate for all compositions in $\mathcal{D}_{J}$. 


\subsubsection{Definition of error}

In order to quantify the accuracy of a model $M_{J}$, we define a normalized error in composition incurred over a reaction sub-step. For a composition in $\mathcal{D}$, here denoted by $\boldsymbol{\Phi}^{D}(0)$, in region $J$ (i.e., $\mathcal{J}\left(\boldsymbol{\Phi}^{D}(0)\right)=J$ ), the composition after a reaction sub-step is

$$
\Phi^{D}(\Delta t)=\int_{0}^{\Delta t} \mathbf{S}\left(\boldsymbol{\Phi}^{D}(t)\right) d t
$$

where $\mathbf{S}$ is the chemical source term given by the detailed mechanism. Using instead the model $M_{J}$, the corresponding reconstructed composition after the reaction sub-step is denoted by $\boldsymbol{\Phi}(\Delta t)$. Based on these two compositions after reaction, we define the model error (for model $M_{J}$ and the initial condition $\boldsymbol{\Phi}^{D}(0)$ ) to be

$$
\varepsilon_{\boldsymbol{\Phi}^{D}(0)} \equiv \frac{\left\|\boldsymbol{\Phi}^{D}(\Delta t)-\boldsymbol{\Phi}(\Delta t)\right\|_{2}}{\left\|\boldsymbol{\Phi}^{D}(\Delta t)\right\|_{2}} .
$$

\subsubsection{Reaction sub-step with reduced modeling}

If the reduced representation contains no locally-important species, so that all non-zero species are present in the model $M_{J}$, then the reaction sub-step using the reduced kinetic model consists of the following steps:

$$
\begin{aligned}
& \text { 1. } \phi_{J}(0)=\mathcal{R}_{F \rightarrow J}\left(\boldsymbol{\Phi}^{D}(0)\right), \\
& \text { 2. } \quad \phi_{J}(\Delta t)=\int_{0}^{\Delta t} \mathbf{S}_{J}\left(\phi_{J}(t)\right) d t, \\
& \text { 3. } \quad \boldsymbol{\Phi}(\Delta t)=\mathcal{R}_{J \rightarrow F}\left(\phi_{J}(\Delta t)\right),
\end{aligned}
$$

where $\mathbf{S}_{J}$ is the chemical source term given by the reduced kinetic model $M_{J}$.

If instead the reduced composition contains locally-significant species (with mass fractions $\widehat{\mathbf{y}}$ ), the treatment is more complicated, because these species are not included in the reduced model. (This is especially relevant when considering PPAC-DRGEP and ISAT combinations, as discussed in sec. 8.1.) Locally-significant species are kinetically inactive (i.e., essentially inert): the only role they play is through their thermal capacity and as acting as third bodies in reactions. We approximate these effects by treating all locally-significant species as being equivalent to an equal mass of a specified inert, which we take to be $N_{2}$.

To implement this treatment in the reaction fractional step described above, after the reduction process (Eq. 34), the mass fraction of $N_{2}$ is increased by $\widehat{y}_{\text {tot }} \equiv \sum \widehat{y}_{i}$, and the value of $\hat{\mathbf{y}}$ in $\phi_{J}(0)$ is set to zero. Then after reaction (Eq. 35), the mass fraction of $N_{2}$ is decreased by $\widehat{y}_{\text {tot }}$ and the original value of $\hat{\mathbf{y}}$ is restored.

It may be noted that this treatment requires that a specified inert must be one of the species represented in each model, although in principle different models could have different species as the designated inert. For the PaSR case considered here, $N_{2}$ is present in every model, so this condition is satisfied. 


\subsubsection{Generation of reduced kinetic models}

For each region $J$, the DRGEP methodology is used to generate a set of models of different sizes and resulting in different errors. Such a pair of model and error is denoted by $\left\{\left.M_{J}\right|_{\varepsilon}, \varepsilon\right\}$ and has the following properties:

1. For every composition $\boldsymbol{\Phi}$ in $\mathcal{D}_{J}$, the model error $\varepsilon_{\boldsymbol{\Phi}}$ is at most $\varepsilon$.

2. All regionally-significant species (as defined below) are retained in the model.

3. Locally-significant species (as defined below) are not retained in the model, but are treated according to the procedure above when evaluating $\varepsilon_{\boldsymbol{\Phi}}$.

4. Subject to 1, 2, and 3, the number of species included in $\left.M_{J}\right|_{\varepsilon}$ is minimum, as is the number of reactions.

A species is deemed to be regionally significant if its mass fraction exceeds $\varepsilon_{\text {sig }}=10^{-3}$ for at least $10 \%$ of the samples in $\mathcal{D}_{J}$, that is, $10 \%$ of the sample compositions contained in region $R_{J}$ of the composition space. It is deemed locally significant for a composition $\mathbf{\Phi}$ if it is not regionally significant, but its mass fraction in $\Phi$ still exceeds $\varepsilon_{\text {sig }}=10^{-3}$. The reason for imposing condition 2 is that the DRGEP methodology eliminates essentially inert species, even if they have large mass fractions, and eliminating such species from the model results in large conversion errors. Condition 3 is a fail-safe mechanism that prevents any one composition with unusually large amount (compared to other compositions in the region) of an inactive species to dominate the model error.

Algorithm 1 details how the DRGEP methodology is used to generate a list of reduced models. Each successive model in the list has smaller size and (usually) larger error.

To improve the ranking ability of DRGEP, sensitivity analysis coefficients can be used to replace $\mathbf{R}^{\mathcal{D}_{J}}$ when $\varepsilon$ becomes larger than a pre-defined value. The sensitivity coefficient of species $A$ is set to 0 if $A$ is INACTIVE, and set to $\varepsilon_{S A}^{A}=\max _{\boldsymbol{\Phi} \in \mathcal{D}_{J}} \varepsilon_{\boldsymbol{\Phi}}^{A}$, where $\varepsilon_{\boldsymbol{\Phi}}^{A}$ is evaluated using the $k$ th model in $\Upsilon$, further reduced by removing species $A$ and all corresponding reactions.

When an adaptive simulation is performed with a specified error tolerance $\varepsilon_{c}$, for every region $J$, the model $M_{J}$ used is the smallest one in the list $\Upsilon$ satisfying $\varepsilon \leqslant \varepsilon_{c}$.

\subsection{Classification}

This and the next two sub-sections describe the operators needed in the adaptive simulation stage, namely Classification, Reconstruction, and Reduction. Note that both Reduction and Reconstruction are also used to evaluate $\varepsilon_{\boldsymbol{\Phi}}$ in the Reduced Modeling algorithm.

The Classification operator $\mathcal{J}$ identifies the region $J$ a composition $\boldsymbol{\Phi}$ belongs to through a simple binary tree search done in the tree formed in the Partitioning stage. First, the value of the classifier is determined as $\mathbf{C}=\mathcal{C}(\boldsymbol{\Phi})$, (Eq. 24). Each node in the tree contains the equation of a cutting hyperplane $H=\{\mathbf{V}, \mathbf{P}\}$, where $\mathbf{V}$ and $\mathbf{P}$ are the normal vector and an anchor point of this hyperplane in the classifying space. Starting from the root of the tree, the scalar product $(\mathbf{C}-\mathbf{P}) \cdot \mathbf{V}$ is computed, and its sign decides which node (or leaf) to access next. The procedure is repeated until a leaf is reached. The index of the region associated with this leaf is returned as $J$. 


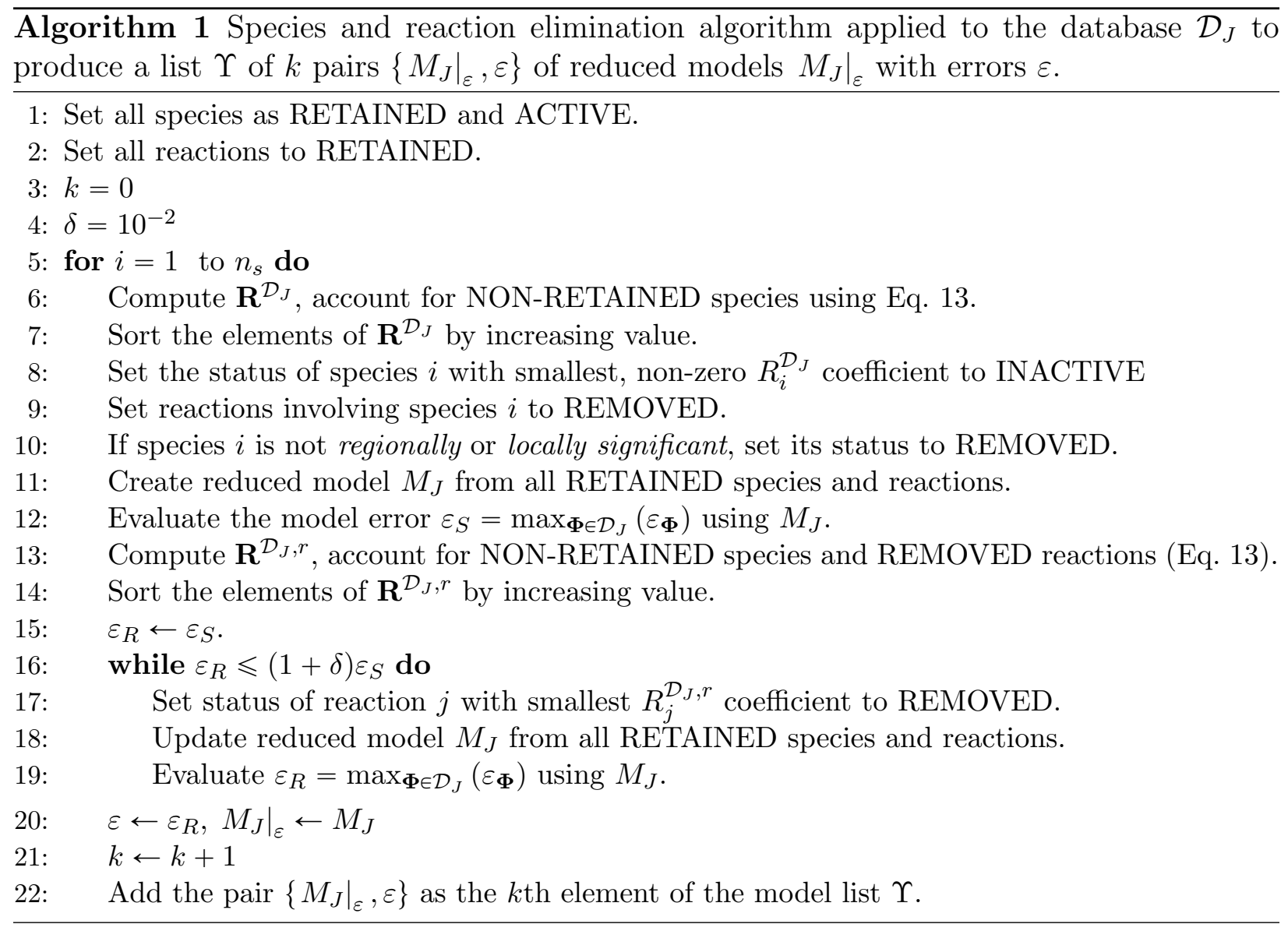




\subsection{Reconstruction}

The $\mathcal{R}_{J \rightarrow F}$ Reconstruction operator reconstructs a full composition vector $\boldsymbol{\Phi}$ from a reduced representation $\phi_{J}$ that involves the reduced set of species $\theta_{J}$ and locally-significant species with mass fractions $\hat{\mathbf{y}}$. While various reconstruction methods have been tested involving various levels of complexity and assumptions, we conclude that the most robust operation is simply to set the state represented by $\boldsymbol{\Phi}$ to be identical to that represented by $\phi_{J}$. This is achieved by: setting all species not represented in $\phi_{J}$ to zero in $\boldsymbol{\Phi}$; setting all other species mass fraction in $\boldsymbol{\Phi}$ to the corresponding mass fractions in $\phi_{J}$; and setting the temperature in $\boldsymbol{\Phi}$ to be the same as that in $\phi_{J}$. This operation conserves enthalpy and species (and therefore also elements and the normalization condition on mass fractions).

\subsection{Reduction}

The last operator that needs to be defined is the $\mathcal{R}_{F \rightarrow J}$ Reduction operator, which performs the reverse of the Reconstruction operator, i.e. taking a full representation and converting it into the reduced representation $\phi_{J}$. A straightforward reduction is adopted here which conceptually involves the following steps:

1. Consider the full representation $\boldsymbol{\Phi}=\{\mathbf{Y}, T\}$.

2. Set $Y_{i}=0$ for every species $i$ that is neither included in the model $M_{J}$ nor is locally significant (which is determined by comparing $Y_{i}$ to $\varepsilon_{\text {sig }}$ ).

3. Rescale the mass fractions $\mathbf{Y}$ so that they sum to unity.

4. Set the mass fractions $\mathbf{y}_{J}$ and $\hat{\mathbf{y}}$ to the corresponding values in $\mathbf{Y}$, and set $T$ to the same temperature as in the full composition to obtain the reduced composition $\phi_{J}=\left\{\mathbf{y}_{J}, \widehat{\mathbf{y}}, T\right\}$.

Because of steps 2 and 3, neither species nor elements are conserved in the reduction. In addition, enthalpy is not conserved (as it could be with a different specification of $\mathrm{T}$ in the reduced representation). The errors resulting from discarding and rescaling species are referred to as conversion errors. We explored variants with improved conservation properties, but none proved superior overall in accuracy and efficiency compared to the simple treatment described above.

\subsection{Summary of inputs to the adaptive chemistry methodology}

We summarize here the inputs to the adaptive chemistry methodology, and give some of their values used in the tests. The required inputs are:

- The detailed chemical mechanism $M_{D}$.

- The targets $\mathcal{T}$ specified for DRGEP, which we take to be fuel, $\mathrm{O}_{2}, \mathrm{OH}, \mathrm{CO}, \mathrm{CO}_{2}$, and heat release.

- The PaSR parameters, including the time step $\Delta t$.

- The number $n_{\mathcal{D}}$ of samples in $\mathcal{D}$, which we take to be $n_{\mathcal{D}}=10,000$. 
- The dimension, $n_{C}$, of the classifying space, which we take to be $n_{C}=10$.

- The number of regions, $N_{R}$, which we vary between 1 and 100.

- The error tolerance, $\varepsilon_{c}$, which we vary over a wide range.

- The mass-fraction threshold for significant species $\varepsilon_{\text {sig }}$, which we take to be $\varepsilon_{\text {sig }}=10^{-3}$.

\section{PaSR test case and composition database}

The configuration chosen to develop and test the adaptive methodology presented above is a non-premixed propane/air piloted PaSR. We consider the detailed mechanism for propane oxidation developed by Curran et al. [46], which consists of $n_{s}=115$ species involved in $n_{r}=1300$ reactions. The PaSR has three inflow streams: pure fuel, pure oxidizer (air), and a pilot stream defined as the burnt products of a stoichiometric propane/air mixture in chemical equilibrium. The pressure is 1 bar throughout. The fuel and air streams are at a temperature of $300 \mathrm{~K}$, while the pilot stream is at the adiabatic flame temperature of $2,272 \mathrm{~K}$. Initially, all particles in the reactor are set to the pilot composition. The number of particles, and hence the total mass, in the reactor remains constant. The particle compositions change in time through reaction and mixing, as described in Section 2, All relevant parameters are included in Table 1 .

The PaSR is run with the above parameters for 10 residence times. We observe a transient state, which depends on the initial conditions, for about the first three residence times, after which the PaSR operates in a statistically stationary state. A sample composition database $\mathcal{D}$ containing $n_{\mathcal{D}}=10,000$ distinct compositions is assembled by randomly sampling the particles in the reactor over the entire duration of the simulation. (The performances of the adaptive chemistry approach is found to be rather insensitive to the number of compositions $n_{\mathcal{D}}$ included in $\mathcal{D}$.)

\section{Results}

In this section we present a quantitative assessment of both the accuracy and efficiency of the adaptive chemistry strategy, as applied to the PaSR simulation.

\subsection{PaSR test runs}

The parameters used in the PaSR simulations are provided in Sec. 6; some of the parameters used in the adaptive methodology are given in Sec. 5.7; and the remainder are given below. A series of PaSR simulations are performed with a broad range of values of the error tolerance, $\varepsilon_{c}$, and for five values of $N_{R}$, namely $N_{R}=1,10,30,60$, and 100 . Consequently, in the pre-processing stage, for each value of $N_{R}$, the partitioning algorithm is applied to determine the $N_{R}$ regions, and for each region the reduced modeling is used to produce the list of models $\left.M_{J}\right|_{\varepsilon}$. Then, prior to a simulation with a specified value of $\varepsilon_{c}$, the smallest models are selected from the list, subject to the condition $\varepsilon \leqslant \varepsilon_{c}$. 
Table 1: Specification of the parameters for the non-premixed propane/air piloted PaSR test case. Species are given as mass fractions. The initial conditions are the same as Stream 1.

\begin{tabular}{|c|c|c|c|}
\hline \multicolumn{4}{|c|}{ Reactor characteristics } \\
\hline Parameters & \multicolumn{2}{|l|}{ Name } & Values \\
\hline Pressure & \multicolumn{2}{|l|}{$p$} & $1 \mathrm{bar}$ \\
\hline Number of particles & \multicolumn{2}{|l|}{$n_{p}$} & 100 \\
\hline Pairing time & \multicolumn{2}{|l|}{$\tau_{\text {pair }}$} & $1 \mathrm{~ms}$ \\
\hline Mixing time & \multicolumn{2}{|l|}{$\tau_{\text {mix }}$} & $1 \mathrm{~ms}$ \\
\hline Residence time & \multicolumn{2}{|l|}{$\tau_{\text {res }}$} & $10 \mathrm{~ms}$ \\
\hline Time step & \multicolumn{2}{|l|}{$\Delta t$} & $0.1 \mathrm{~ms}$ \\
\hline \multicolumn{4}{|c|}{ Initial and Inflow conditions (Species data are mass fractions) } \\
\hline Parameters & Stream 1 & Stream 2 & Stream 3 \\
\hline Normalized mass flow rates & 0.05 & 0.893 & 0.057 \\
\hline Temperature & $2272 \mathrm{~K}$ & $300 \mathrm{~K}$ & $300 \mathrm{~K}$ \\
\hline $\mathrm{C}_{3} \mathrm{H}_{8}$ & 0.0 & 0.0 & 1.0 \\
\hline $\mathrm{N}_{2}$ & $7.2085 \times 10^{-1}$ & 0.767 & 0.0 \\
\hline $\mathrm{O}_{2}$ & $7.6607 \times 10^{-3}$ & 0.233 & 0.0 \\
\hline $\mathrm{CO}_{2}$ & $1.6146 \times 10^{-1}$ & 0.0 & 0.0 \\
\hline $\mathrm{H}_{2} \mathrm{O}$ & $9.5159 \times 10^{-2}$ & 0.0 & 0.0 \\
\hline $\mathrm{CO}$ & $1.2162 \times 10^{-2}$ & 0.0 & 0.0 \\
\hline $\mathrm{OH}$ & $2.2616 \times 10^{-3}$ & 0.0 & 0.0 \\
\hline $\mathrm{H}_{2}$ & $2.2880 \times 10^{-4}$ & 0.0 & 0.0 \\
\hline $\mathrm{O}$ & $1.9634 \times 10^{-4}$ & 0.0 & 0.0 \\
\hline $\mathrm{H}$ & $1.6928 \times 10^{-5}$ & 0.0 & 0.0 \\
\hline $\mathrm{HO}_{2}$ & $8.0954 \times 10^{-7}$ & 0.0 & 0.0 \\
\hline $\mathrm{H}_{2} \mathrm{O}_{2}$ & $6.1000 \times 10^{-8}$ & 0.0 & 0.0 \\
\hline $\mathrm{HCO}$ & $1.2371 \times 10^{-9}$ & 0.0 & 0.0 \\
\hline $\mathrm{CH}_{2} \mathrm{O}$ & $1.6891 \times 10^{-11}$ & 0.0 & 0.0 \\
\hline
\end{tabular}

The adaptive PaSR simulations are then carried out according to the algorithm described in Section 3 and illustrated in Fig. 3. A (non-adaptive) PaSR simulation is also performed using the detailed chemical mechanism $M_{D}$ and the full representation to serve as reference for error measurements. Quantities extracted from the tests with the adaptive strategy and with detailed chemistry are indicated with the superscripts "A" and "D", respectively.

It is found that the results obtained for the degenerate case $N_{R}=1$ are qualitatively different from those obtained with larger values of $N_{R}$. This case is degenerate in that the adaptive methodology is in fact not adaptive. Henceforth we therefore refer to this as the non-adaptive case.

In order to create a fair test of the adaptive method, the seed that generates the pseudorandom numbers used in the PaSR simulations is changed from that used to create the sample database $\mathcal{D}$. Therefore the PaSR particles follow trajectories in the same areas of the composition space, but they are not strictly identical to those obtained in the pre-processing 
stage. Particle evolutions are recorded over a total time $\tau$ equal to 10 residence times, corresponding to $n_{t}=1,000$ time steps.

\subsection{Definition of error}

Errors are measured for species mass fractions and temperature. Rather than comparing reactor statistics, such as mean values, we opt for a more stringent test of our approach by comparing individual particle trajectories obtained in the detailed and adaptive PaSR simulations. We choose the following integrated error measure, written here for any quantity $X$ (species mass fraction or temperature):

$$
\varepsilon_{X}=\frac{\sum_{k=1}^{n_{t}} \sum_{n=1}^{n_{p}}\left|X_{k}^{(n), A}-X_{k}^{(n), D}\right|}{\sum_{k=1}^{n_{t}} \sum_{n=1}^{n_{p}}\left|X_{k}^{(n), D}\right|} .
$$

In the above equation, $X_{k}^{(n), A}$ and $X_{k}^{(n), D}$ denote the value of quantity $X$ for particle $n$ on time step $k$ of a PaSR simulation with adaptive chemistry and detailed chemistry, respectively.

\subsection{Control of errors}

Figure 7 shows the incurred error in temperature, $\varepsilon_{T}$, as a function of the specified error tolerance $\varepsilon_{c}$ for each of the five values of $N_{R}$. As may be seen, the adaptive strategy is successful in controlling the error, in that, for each value of $N_{R}$, the incurred error $\varepsilon_{T}$ decreases monotonically as the error tolerance $\varepsilon_{c}$ is decreased, until a floor is reached at the very low value of $10^{-6}$. In a turbulent combustion calculation, an error of $1 \%$ in species mass fractions and $5 \mathrm{~K}$ in temperature (i.e., $\varepsilon_{T} \approx 3 \times 10^{-3}$ ) is generally regarded as more than acceptable.

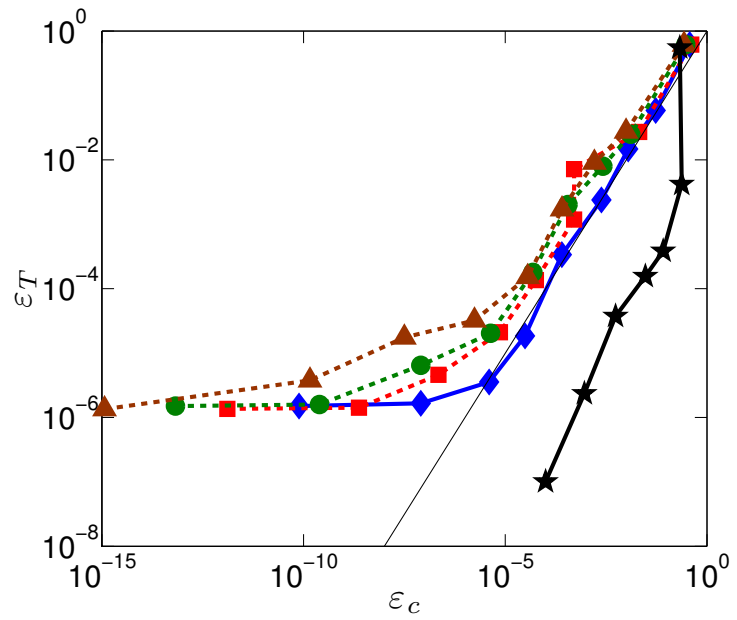

Figure 7: The incurred error in temperature, $\varepsilon_{T}$, vs. the specified error tolerance, $\varepsilon_{c}$, in PaSR simulations. Comparison between the non-adaptive case, $N_{R}=1(\star)$ and adaptive cases with $N_{\mathcal{R}}=10(\diamond), 30(\bullet), 60(\bullet)$, and $100(\Delta)$. The unit slope line (thin black line) is added for reference. 
We recall that there are two sources of error. The first is in the reduced kinetic model, and this is directly controlled by $\varepsilon_{c}$. The second is the conversion error. This is controlled indirectly by $\varepsilon_{c}$, since the smaller $\varepsilon_{c}$, the larger the reduced models, and hence the smaller the number (and magnitude) of species omitted from the models. As may be seen from Fig. 7 , for a fixed value of the error tolerance $\varepsilon_{c}$, the incurred error $\varepsilon_{T}$ increases with increasing $N_{R}$. This is likely due to a combination of two effects. First, the larger $N_{R}$, the more crossings there are likely to be from one region to another, and hence more conversions are performed. Second, the larger $N_{R}$, the smaller (on average) the regions, and hence fewer species are needed to represent the more specialized chemistry, and hence there are more species omitted from the models.

It is the incurred error (not the error tolerance) that is of consequence, and in comparing two approaches or variants, this should be done at fixed incurred error, not at fixed error tolerance. Consequently, henceforth, we use $\varepsilon_{T}$ to parameterize the error, and we plot quantities of interest against $\varepsilon_{T}$ rather than $\varepsilon_{c}$.

Figure 8 shows the incurred error for different species mass fractions plotted against the incurred error in temperature for the adaptive case with $N_{R}=30$. As may be seen, for $\mathrm{CO}_{2}, \mathrm{C}_{3} \mathrm{H}_{8}$ and $\mathrm{H}_{2} \mathrm{O}$, the species errors are very close to the temperature error, whereas the error for $\mathrm{CO}$ is about 5 times that for $T$. Results for different values of $N_{R}$ and for different species are similar to those shown in Fig. 8. The important observation is that $\varepsilon_{T}$ provides a good characterization of all of the errors.

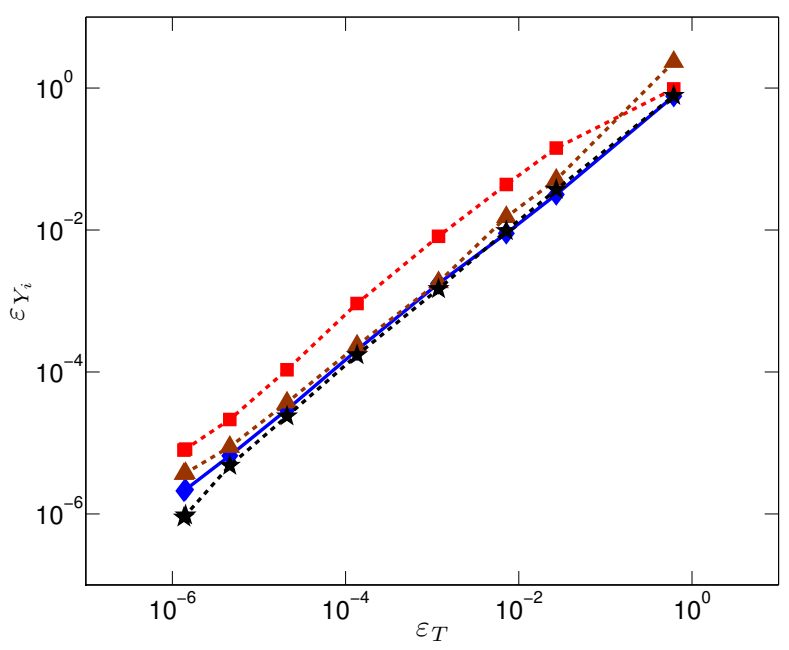

Figure 8: Incurred error in species $\varepsilon_{Y_{i}}$ vs. incurred error in temperature $\varepsilon_{T}$ for $N_{R}=30$. Species are: $\mathrm{CO}_{2}$ $(\diamond), C O(\bullet), \mathrm{C}_{3} \mathrm{H}_{8}(\bullet), \mathrm{H}_{2} \mathrm{O}(\star)$.

To help relate the error levels as defined in Eq. 37 to actual temperature changes, in Fig. 9 (top), the mean reactor temperature is plotted over three residence times for the detailed and adaptive simulations with $\varepsilon_{T}=10^{-3}$ and $N_{R}=30$. As may be seen, the two curves are essentially indistinguishable, and so we plot their difference in Fig. 9 (bottom). Overall, the 
maximum temperature discrepancies are around $2.5 \mathrm{~K}$ out of $1650 \mathrm{~K}$, and more importantly, these discrepancies are found not to increase in any significant manner over time, an essential property for the adaptive strategy to be viable in large-scale simulations. This behavior has been observed consistently for all quantities discussed below.
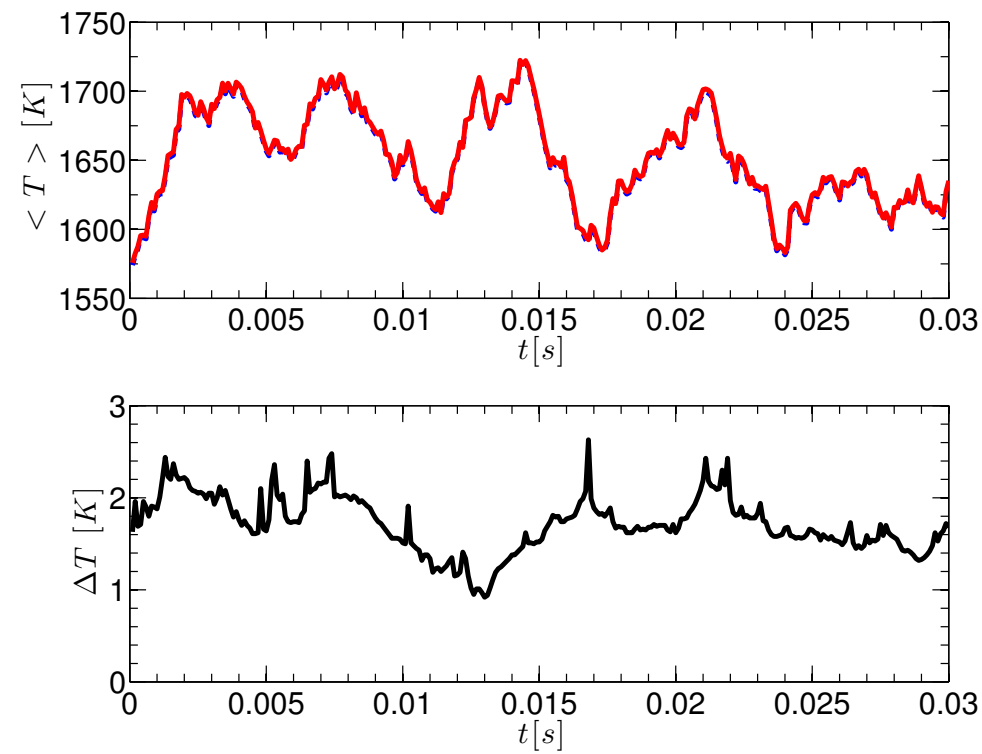

Figure 9: Top: Evolution of the mean temperature in the PaSR for the detailed $\left(\left\langle T^{D}\right\rangle\right.$, red solid line) and adaptive $\left(\left\langle T^{A}\right\rangle\right.$, blue dashed line) simulations using $\varepsilon_{T}=10^{-3}$ and $N_{R}=30$. (The two lines are indistinguishable.) Bottom: the absolute difference $\Delta T=\left|\left\langle T^{A}\right\rangle-\left\langle T^{D}\right\rangle\right|$ in the mean temperature between the two simulations.

\subsection{Computer time}

The PaSR simulations are performed in parallel on a Beowulf cluster based on Nehalem $\mathrm{X}$ series processors, QDR Infiniband, and 2 GB of RAM per core. For the simulation using detailed chemistry, the wall clock time needed to reach $t=10 \tau_{\text {res }}$ on 12 cores is $4020 \mathrm{~s}$. For a given number of regions $N_{R}$, the partitioning, reduction, and model evaluation over the range of incurred errors shown in Fig. 8 require on average $10^{3}$ CPU hours. This is but a small fraction of the typical cost of LES/PDF simulations, which can quickly reach upward of $10^{5}$ CPU hours even for simple flames. This cost therefore appears very reasonable, especially since the computational overhead due to the adaptive treatment of the chemistry at run time in the PPAC approach is negligible.

We define the relative time $t_{\text {rel }}$ of an adaptive simulation as its CPU time divided by that of the simulation with detailed chemistry. Figure 10 shows the relative time $t_{\text {rel }}$ as a function of the incurred temperature error for the different values of $N_{R}$. It may be observed that, as expected, all the simulations using reduced models require less CPU time than those using the detailed mechanism (i.e., $t_{\text {rel }}$ is less than unity). Furthermore, the adaptive cases 
$\left(N_{R}>1\right)$ require, typically, just half the time of the non-adaptive case $\left(N_{R}=1\right)$. At an incurred error of $\varepsilon_{T}=3 \times 10^{-3}$, the $\mathrm{CPU}$ time required for the adaptive cases is about $15 \%$ of that for the detailed mechanism, and about $55 \%$ of that for the non-adaptive case. In general, the CPU time decreases weakly with $N_{R}$, for $N_{R} \geqslant 10$.

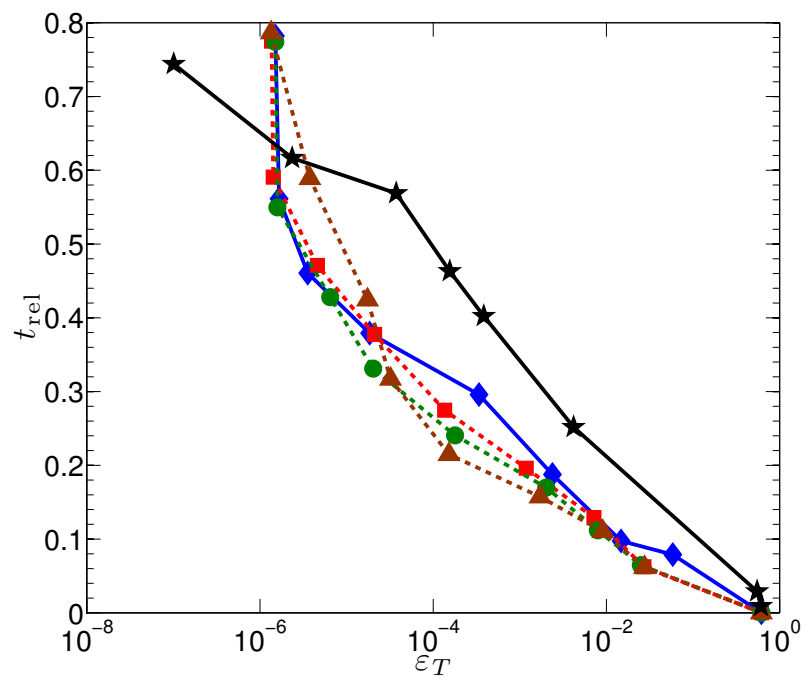

Figure 10: Relative CPU time (adaptive chemistry to detailed chemistry) vs. the incurred temperature error. Comparison between the non-adaptive case, $N_{R}=1,(\star)$ and adaptive cases with $N_{R}=10(\diamond), 30(\bullet), 60$ $(\bullet)$, and $100(\triangleleft)$.

The above comparisons are much in favor of the adaptive approach, even in a PaSR with pair-wise mixing. It is expected that its benefits will be even more pronounced in a turbulent combustion calculation, since the portion of particles carrying inerts or burnt compositions, described efficiently with few variables, is much higher than in a PaSR.

The results presented so far use DRGEP combined species and reaction elimination, as described in Section 5.3. While already effective, the standard DRGEP approach can be complemented by sensitivity analysis, to refine the ranking of species identified as of intermediate importance by DRGEP. Some incremental gain in CPU cost is observed when switching to sensitivity coefficients in lieu of DRGEP coefficients for $\varepsilon_{c}$ larger than $10^{-8}$, as shown in Fig. 11. In view of these results, the cost vs. benefit of using expensive sensitivity analysis methods to further decrease the complexity of the region-specific reduced representations and models for larger reference mechanisms warrants further investigation.

\subsection{Computer memory}

Another quantity of interest relates to the storage requirements of the PaSR simulations. This can be measured by the relative number of species, $n_{\text {rel }}$, defined as the number of species in the reduced models used (averaged over all particles and time steps) divided by the number 


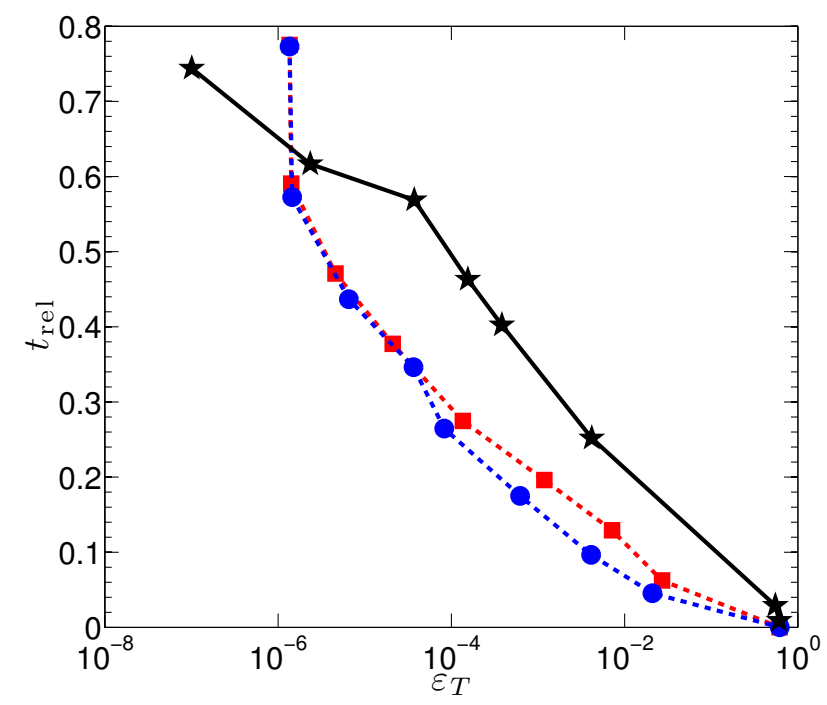

Figure 11: Relative CPU cost vs. the incurred temperature error $\varepsilon_{T}$ for $N_{R}=30$. Comparison between reduced modeling performed using standard DRGEP coefficients ( $\bullet$ ), and reduced modeling performed using standard DRGEP coefficients up to $\varepsilon_{c}=10^{-8}$, and sensitivity coefficients thereafter $(\bullet)$. The non-adaptive results of Fig. 10, which are not using sensitivity analysis, are added here for reference.

of species in the detailed mechanism, $n_{s}$ :

$$
n_{\mathrm{rel}} \equiv \frac{1}{n_{t} n_{p} n_{s}} \sum_{k=1}^{n_{t}} \sum_{n=1}^{n_{p}} n_{s, k}^{(n), A}
$$

where $n_{s, k}^{(n), A}$ is the number of species in the reduced model used for particle $n$ on time step $k$.

Figure 12 shows $n_{\text {rel }}$ as a function of the incurred error $\varepsilon_{T}$ for each value of $N_{R}$. This shows, for any specified incurred temperature error in the adaptive PaSR simulations, the degree of reduction that can be achieved by the method. All curves are monotonically decreasing with increasing $\varepsilon_{T}$, from values very close to unity, corresponding to very low error levels at one extreme (in which the reduced models are very close to the detailed model), to large errors and very small reduced models at the other extreme. A clear difference is observed between the non-adaptive case $\left(N_{R}=1\right)$ and the adaptive cases, with the number of species retained to achieve a specified error tolerance being up to a factor of two lower for the adaptive cases (compared to the non-adaptive case). Consider $\varepsilon_{T}=10^{-3}$ as a typical desired error level: in this case of the order of $30 \%$ of the species are needed (on average) in the adaptive simulations, compared to roughly $60 \%$ for the non-adaptive case $\left(N_{R}=1\right)$. Some minor reduction in $n_{\text {rel }}$ is observed when increasing the number of regions $N_{R}$ above 10. However, in the range of error typically of interest $\left(10^{-4}\right.$ to $\left.10^{-2}\right)$, cases with $N_{R} \geqslant 30$ can be considered equivalent. Unless stated otherwise, $N_{R}=30$ is therefore used in all cases below. 


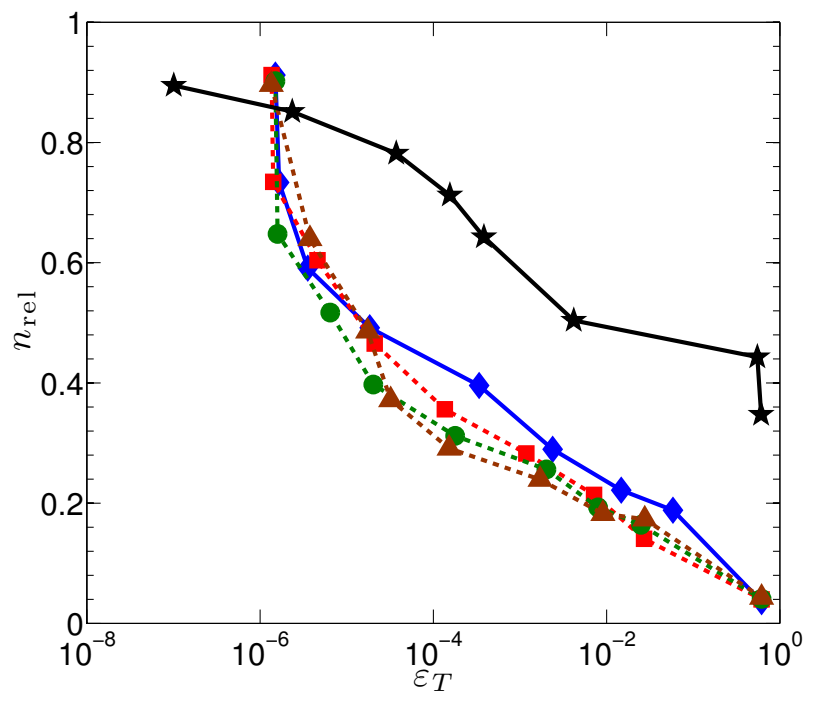

Figure 12: Relative number of species $n_{\text {rel }}$ (adaptive to detailed) $v s$. the incurred error in temperature $\varepsilon_{T}$. Comparison between the the non-adaptive case, $N_{R}=1(\star)$ and adaptive cases with $N_{R}=10(\bullet), 30(\star), 60$ $(\bullet)$, and $100(\Delta)$.

\subsection{Conservation properties}

In the detailed PaSR simulation, over the mixing and reaction fractional steps, when summed (or averaged) over all particles, both enthalpy and the mass fractions of elements are conserved. However, in the adaptive simulations, the reduction operator $\mathcal{R}_{F \rightarrow J}$ (see Sec. 5.6 is not conservative. For elements, this is because some species mass fractions are set to zero, and then the mass fractions are rescaled to sum to unity. For the enthalpy, it is because the reduction is performed as constant temperature rather than at constant enthalpy. However, it is expected that the resulting conservation errors are small, since if a species is not part of a representation, its mass fraction is small by construction (certainly less than $\left.\varepsilon_{\text {sig }}\right)$. To test this hypothesis, the total enthalpy and element mass fractions are followed over time in adaptive PaSR simulations using $N_{R}=30$ and a range of error tolerances. The normalized conservation error $\hat{\varepsilon}_{X}$ for a conserved quantity $X$ is defined as:

$$
\widehat{\varepsilon}_{X}=\frac{1}{n_{p}} \frac{1}{n_{t}} \sum_{k=1}^{n_{t}}\left|\frac{\sum_{n=1}^{n_{p}}\left(X_{k+}^{(n)}-X_{k}^{(n)}\right)}{\sum_{n=1}^{n_{p}} X_{k}^{(n)}}\right|,
$$

where, for particle $n$ on time step $k, X_{k}^{(n)}$ and $X_{k+}^{(n)}$ denote the value of $X$ before and after the mixing and reaction fractional steps, respectively. Conservation errors for enthalpy and element mass fractions are plotted in Fig. 13 as functions of the temperature error $\varepsilon_{T}$. Note that as mentioned above, those conservation errors are integrated over the full simulation, and no significant increase of the instantaneous values is observed over time. When the number of species retained decreases (leading to higher conversion errors) and temperature 
error increases, errors for the conserved quantities remain below $10^{-5}$ for all cases and quantities of interest, indicating satisfactory treatment of particle conversion between various representations.

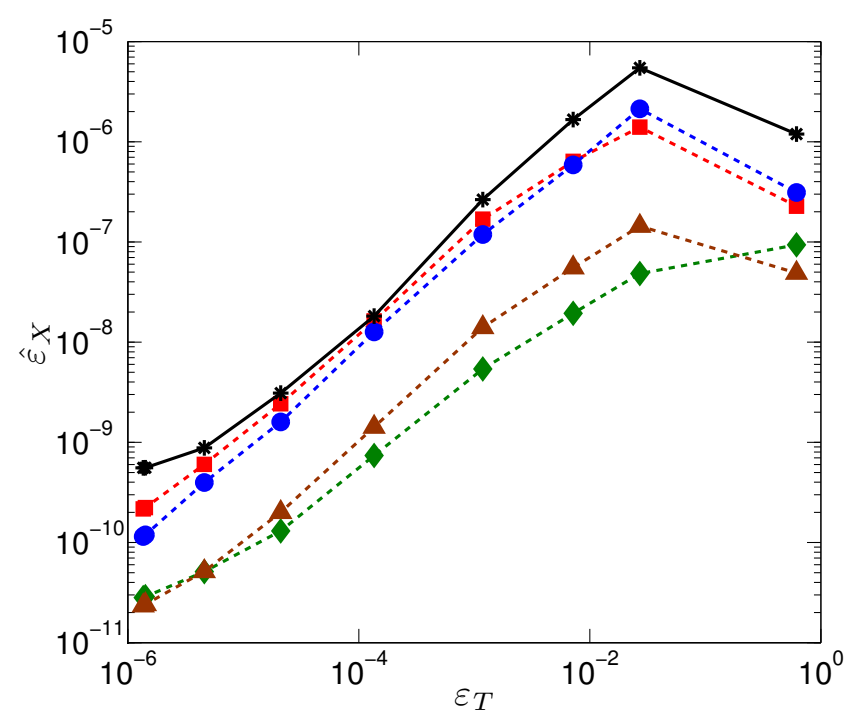

Figure 13: Conservation errors as function of the temperature error in the adaptive PaSR simulation: Enthalpy $(*$, solid line), mass fraction of carbon atoms (๑), hydrogen atoms $(\bullet)$, oxygen atoms $(\diamond)$, and nitrogen atoms $(\boldsymbol{\Delta})$.

\subsection{Regions accessed and model utilization}

A major assumption at the core of the adaptive strategy is that the sample composition database $\mathcal{D}$ is representative of the particle compositions that occur in the adaptive simulations. To confirm the validity of this assumption a posteriori, the distribution of compositions in $\mathcal{D}$ across regions is compared to the frequency at which particles are classified into those same regions during the adaptive PaSR simulation. Recall that, since pairing in the PaSR is a random process, we do not recover the database compositions during the adaptive simulation. Results are shown in Fig. 14, demonstrating similar region access frequency between pre-processing and adaptive simulation stages. Figure 15 illustrates the trajectory of a particular particle in $\mathbb{C}$ space, by providing side-to-side the temperature evolution and the index of the region the particle belongs to at time $t$. Comparing both plots shows that changes in pairing partners are often followed by a change in region (i.e. model switches often occurs a few time steps after discontinuities in temperature gradients) but particles can also switch to a different representation outside of those discrete events.

While the above result brings confidence that $\mathcal{D}$ contains the needed relevant kinetic information, being able to predict model utilization also has important implications for large-scale simulations, especially in terms of load balancing and coupling with tabulation methods such as ISAT. 


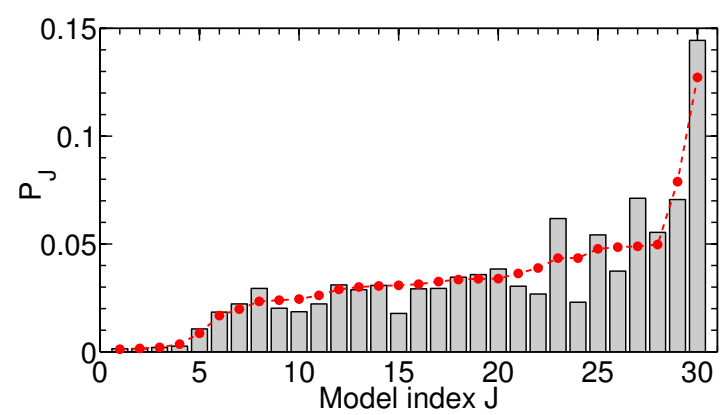

Figure 14: Fraction of compositions in $\mathcal{D}$ included in Region $J(\bullet)$ compared to the overall probability $P_{J}$ of a particle to be classified as belonging to region $J$ (bars) during the adaptive simulation stage. Regions are sorted according to the fraction of database compositions they contain.

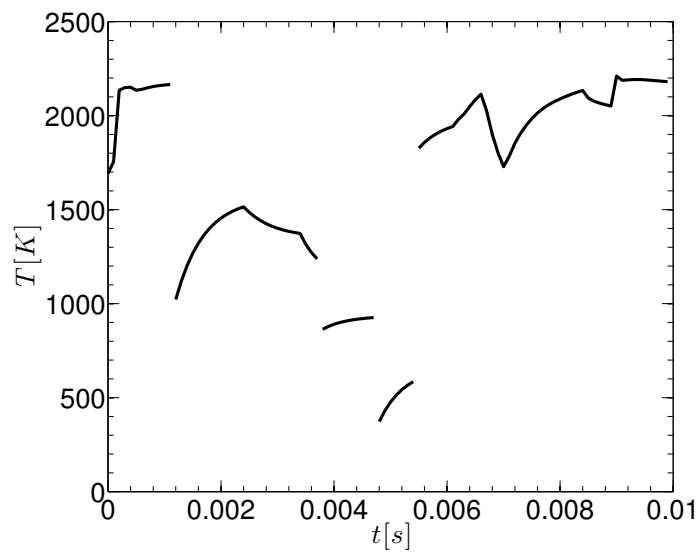

(a) Particle temperature evolution.

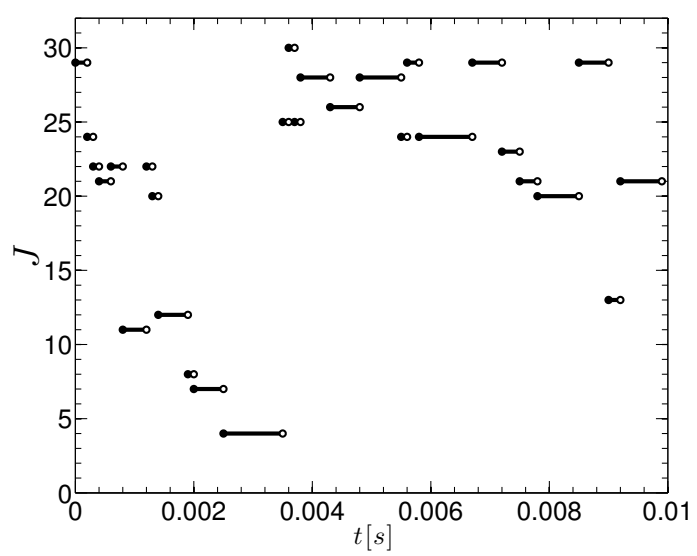

(b) Index of region in $\mathbb{C}$ space returned by the classification operator.

Figure 15: Temporal evolution of temperature $T$ (left) and region index $J$ (right) for one particle in an adaptive simulation with $N_{R}=30$ in a non-premixed propane/air PaSR. Jumps in $T$ are due to the inflow/outflow events.

\section{Discussion}

In this section we discuss some possible improvements and extensions to the adaptive methodology, and then consider future applications of the method in LES/PDF simulations.

\subsection{Improvements and extensions}

It is evident that the number of regions $N_{R}$ is not a computational limitation, in that of order tens are sufficient, whereas of order hundreds could be handled without difficulty in computations. One can therefore consider local improvements to the partitioning: if a region is found to require a large reduced kinetic mechanism, it can (recursively) be split into two smaller regions, provided that (as expected) these two regions have smaller models.

The most obvious and beneficial extension to the methodology is to combine it with in situ adaptive simulation (ISAT) [23, 24]. For each region $J$, a separate ISAT table can be 
used to perform the reaction fractional step much more efficiently than by integrating the kinetic equations. In ISAT, both the work to retrieve from the table, and the storage needed per table entry scale as the square of the number of species. Furthermore, the performance of ISAT is found to degrade significantly beyond about 50 species. Hence, there is great benefit in having $N_{R}$ tables for reduced representation with relatively few species (e.g., $n_{s}^{J}<50$ ), compared to one table involving hundreds or thousands of species.

It is straightforward to combine the adaptive methodology with ISAT in serial computations (or to use purely local processing [43] in parallel simulations); and the fact that there are many tables opens up new possibilities for efficient parallel implementations.

\subsection{Use in $L E S / P D F$ simulations}

For the PaSR test case used here, it is guaranteed that the database $\mathcal{D}$ of compositions generated in the pre-processing stage are representative of those in the adaptive simulations, since both stages use statistically-identical PaSR simulations. For an adaptive LES/PDF simulation, we propose two sub-stages in the pre-processing stage in order to generate a suitably representative database. In the first sub-stage, one or more PaSR simulations are performed with conditions representative of the LES/PDF simulation to generate the first database $\mathcal{D}_{1}$, and the reduced models are generated with a reasonably small error tolerance. Then, using the adaptive method, a coarse LES/PDF simulation is performed, for example, with double the grid spacing in each direction, and half the number of particles and simulation time compared to the full simulation, resulting is approximately $1 / 64$ of the cost of the full simulation. This coarse LES/PDF is used to generate the representative database $\mathcal{D}$ used subsequently in the adaptive strategy. (A small error tolerance is used in the first substage to ensure that all needed species are included in $\mathcal{D}_{1}$.) When considering large detailed mechanisms (with more than, say, 300 species) it is impracticable to perform even a coarse LES/PDF with the detailed mechanism. This is why a PaSR is used in the first sub-stage. Note that further investigation is required to evaluate the relative costs involved in such a process, especially when compared to on-the-fly adaptive techniques. Yet, this provides a path forward to systematically handle simulations with very large kinetic mechanisms, which would not be otherwise feasible.

In contrast to on-the-fly adaptive chemistry methodologies, in the present method, the errors are not controlled during the simulation, but are instead controlled as part of the preprocessing stage. Their control during the adaptive simulation depends on the database $\mathcal{D}$ used in the pre-processing stage being completely representative. It is, therefore, desirable to be able to measure the errors during the adaptive simulation, and hence to verify that they are indeed controlled (and possibly to invoke a correction strategy if they are not). This can be done with negligible computational penalty (as it is in ISAT [24]) by randomly sampling a small fraction (e.g., 10 ${ }^{-4}$ ) of the particle compositions prior to the reaction fractional step,

and comparing the reaction mappings obtained with the reduced model and the detailed mechanism. 


\section{Conclusions}

A novel adaptive strategy specifically tailored for particle PDF methods to simulate turbulent combustion systems has been presented.

Rather than reducing the chemistry on-the-fly during the combustion simulation, a set of reduced representations and reduced kinetic models is assembled a priori, which are assigned adaptively to each individual particle during the simulation. The approach includes the following major characteristics:

- In the pre-processing stage: a database $\mathcal{D}$ of representative compositions is generated; a classifying space is defined and suitably partitioned into $N_{R}$ regions, for each of which DRGEP is used to generate a locally-accurate reduced kinetic mechanism $M_{J}$, much smaller than the detailed mechanism; and the particle composition in region $R_{J}$ is represented by a reduced representation $\phi_{J}$.

- In the adaptive simulation stage, for each PDF particle: a classification algorithm based on a low-dimensional binary tree is used to identify the region $J$ the particle's composition belongs to; the reduced kinetic model $M_{J}$ is used to advance the particle composition in the reaction fractional step; and, the reduced representation of a particle composition can be adaptively chosen and converted as it moves from one region to another.

Based on these characteristics, the methodology is referred to as Pre-Partitioned Adaptive Chemistry using DRGEP, or PPAC-DRGEP.

The performance of the adaptive methodology has been demonstrated for a propane/air non-premixed PaSR. The results show that the incurred errors are well controlled by the specified error tolerance, and can be reduced well below acceptable levels. Even though the methodology does not exactly conserve elements or enthalpy, nevertheless, the error in theses conserved quantities is very small. Typically, the CPU time using the adaptive strategy is $15 \%$ of that using the detailed mechanism; and $50 \%$ of that using a single reduced mechanism. On average, only $30 \%$ of the species are included in the reduced models. These computational gains are expected to be much larger in PDF simulations and with larger detailed mechanisms.

As discussed in Sec. 8, in future work, the adaptive methodology will be combined with ISAT and used in LES/PDF simulations of turbulent flames.

\section{Acknowledgement}

This material is based upon work supported by the U.S. Department of Energy Office of Science, Office of Basic Energy Sciences under Award Number DE-FG02-90ER14128.

\section{Appendix}

This appendix details the algorithm used to accomplish the partitioning step depicted in Fig. 5c. We consider a set of compositions $\mathcal{D}_{m}$, divided into two complementary subsets $\mathcal{D}_{m, L}^{*}$ 
and $\mathcal{D}_{m, R}^{*}$, of size $n_{L}$ and $n_{R}$ respectively. $\mathcal{D}_{m, L}^{*}$ and $\mathcal{D}_{m, R}^{*}$ have been obtained by minimization in $\mathbb{R}$ space of $c_{m}$ along the first principal component direction of the normalized DRGEP coefficient vectors $\mathbf{R}^{\boldsymbol{\Phi}}$, for all $\boldsymbol{\Phi} \in \mathcal{D}$. We want to identify an hyperplane $H=\{\mathbf{V}, \mathbf{P}\}$ in $\mathbb{C}$ such that

$$
\begin{array}{ll}
\left(\mathbf{C}_{\boldsymbol{\Phi}}-\mathbf{P}\right) \cdot \mathbf{V}>0 & \text { for } \boldsymbol{\Phi} \in \mathcal{D}_{m, L}^{*}, \text { and } \\
\left(\mathbf{C}_{\boldsymbol{\Phi}}-\mathbf{P}\right) \cdot \mathbf{V}<0 & \text { for } \boldsymbol{\Phi} \in \mathcal{D}_{m, R}^{*},
\end{array}
$$

where $\mathbf{C}_{\boldsymbol{\Phi}}=\mathcal{C}(\boldsymbol{\Phi})$ (Eq. 24). Due to the non-linear implicit transformation between $\mathbb{C}$ and $\mathbb{R}$ spaces, the existence of an hyperplane in $\mathbb{C}$ that satisfies those inequalities for all $\Phi \in \mathcal{D}_{m}$ is not guaranteed. Instead, a pragmatic approach based on covariance ellipsoids is used. The steps are as follows (all operations are made in $\mathbb{C}$-space):

1. Form the means $\mathbf{m}_{L}$ and $\mathbf{m}_{R}$ of $\mathbf{C}_{\boldsymbol{\Phi}}$ vectors from compositions contained in $\mathcal{D}_{m, L}^{*}$ and $\mathcal{D}_{m, R}^{*}$, respectively.

2. Form the ellipsoid $E_{L}$ defined by

$$
E_{L} \equiv\left\{\mathbf{C} \mid\left(\mathbf{C}-\mathbf{m}_{L}\right)^{T} C_{L}^{-1}\left(\mathbf{C}-\mathbf{m}_{L}\right) \leqslant s^{2}\right\}
$$

where $\mathbf{C} \in \mathbb{C}, C_{L}$ is the covariance matrix of all $\mathbf{C}_{\boldsymbol{\Phi}}$ from $\mathcal{D}_{m, L}^{*}$, and $s$ determines the size of the ellipsoid. $E_{R}$ is similarly defined. This step is illustrated in Fig. 16a.

3. Determine the smallest value of $s$ at which $E_{L}$ and $E_{R}$ intersect. This is achieved using the ELL_LIB library developed by Pope [47].

4. The normal vector $\mathbf{V}$ of the cutting hyperplane $H$ is taken to be that of the plane tangent to both ellipsoids at the point of intersection (Fig. 16b).

5. The anchor point $\mathbf{P}$ is chosen along the direction of $\mathbf{V}$ to minimize the kinetic inhomogeneity $c_{m}$ of the resulting $\mathcal{D}_{m, L}$ and $\mathcal{D}_{m, R}$, following the same procedure as that in Fig. 5b, ii).

\section{References}

[1] T. Lu, C. K. Law, Toward accommodating realistic fuel chemistry in large-scale computations, Prog. Energy Combust. Sci. 35 (2009) 192-215.

[2] O. Herbinet, W. J. Pitz, C. K. Westbrook, Detailed chemical kinetic oxidation mechanism for a biodiesel surrogate, Combust. Flame 154 (2008) 507-528.

[3] R. W. Grout, A. Gruber, C. S. Yoo, J. H. Chen, Direct numerical simulation of flame stabilization downstream of a transverse fuel jet in cross-flow, Proc. Combust. Inst. 158 (2011) 2113-2127.

[4] V. Hiremath, S. R. Lantz, H. Wang, S. B. Pope, Large-scale parallel simulations of turbulent combustion using combined dimension reduction and tabulation of chemistry, Proc. Combust. Inst. 34 (1) (2013) $205-215$. 


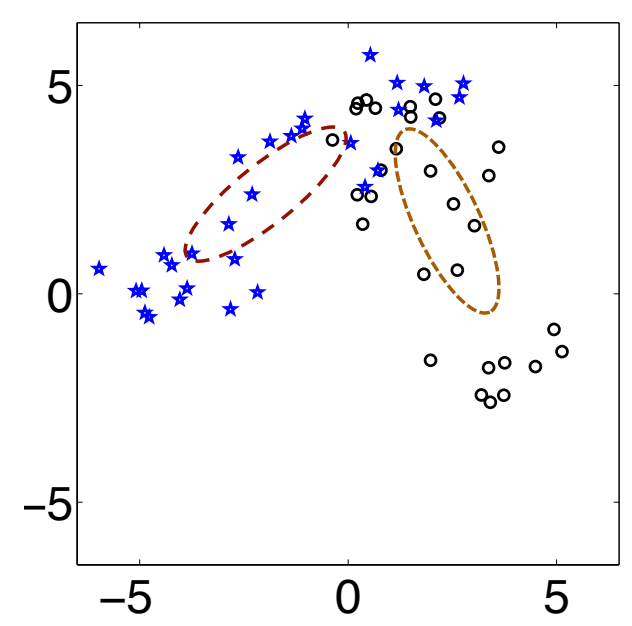

(a) Initial subsets $\mathcal{D}_{m, L}^{*}$ (blue stars) and $\mathcal{D}_{m, R}^{*}$ (open black circles), along with ellipsoids $E_{L}$ and $E_{R}$.

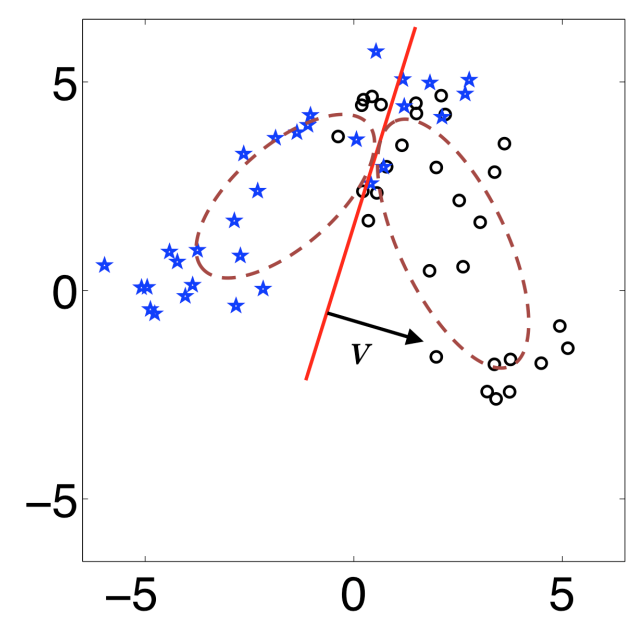

(b) The size $s$ of $E_{L}$ and $E_{R}$ are adjusted so that they intersect at one point. The hyperplane normal vector is chosen as that normal to the tangent plane.

Figure 16: Two-dimensional illustration of the use of ellipsoids to identify the cutting hyperplane $H$ in C-space.

[5] N. Peters, Turbulent Combustion, Cambridge University Press, 2000.

[6] C. D. Pierce, P. Moin, Progress-variable approach for large eddy simulation of turbulent combustion, Tech. Rep. TF80, Flow Physics and Computation Division, Dept. Mech. Eng., Stanford University (2001).

[7] T. Lu, C. K. Law, Linear time reduction of large kinetic mechanisms with directed relation graph: $n$-heptane and iso-octane, Combust. Flame 144 (2006) 24-36.

[8] T. Lu, C. K. Law, On the Applicability of Directed Relation Graphs to the Reduction of Reaction Mechanisms, Combust. Flame 146 (2006) 472-483.

[9] P. Pepiot, H. Pitsch, An efficient error-propagation-based reduction method for large chemical kinetic mechanisms, Combust. Flame 154 (2008) 67-81.

[10] K. E. Niemeyer, C. J. Sung, M. P. Raju, Skeletal mechanism generation for surrogate fuels using directed relation graph with error propagation and sensitivity analysis, Combust. Flame 157 (9) (2010) 1760-1770.

[11] M. Valorani, F. Creta, D. A. Goussis, J. C. Lee, H. N. Najm, An automatic procedure for the simplification of chemical kinetic mechanisms based on CSP, Combust. Flame 146 (1-2) (2006) 29-51.

[12] T. Nagy, T. Turanyi, Reduction of very large reaction mechanisms using methods based on simulation error minimization, Combust. Flame 156 (2009) 417-428. 
[13] W. Sun, Z. Chen, X. Gou, Y. Ju, A path flux analysis method for the reduction of detailed chemical kinetic mechanisms, Combust. Flame 157 (2010) 1298-1307.

[14] P. Pepiot, H. Pitsch, A chemical lumping method for the reduction of large chemical kinetic mechanisms, Combust. Theory Model. 12 (2008) 1089-1108.

[15] S. S. Ahmed, F. Mauss, G. Moréac, T. Zeuch, A comprehensive and compact $n$-heptane oxidation model derived using chemical lumping, Phys. Chem. Chem. Phys. 9 (2007) $1107-1126$.

[16] H. Huang, M. Fairweather, J. F. Griffiths, A. S. Tomlin, R. B. Brad, A systematic lumping approach for the reduction of comprehensive kinetic models, Proc. Combust. Inst. 30 (2005) 1309-1316.

[17] M. Bodenstein, S. C. Lind, Geschwindigkeit der bildung des bromwasserstoffs aus seinen elementen, Z. Phys. Chem. 57 (1906) 168.

[18] J. Y. Chen, A general procedure for constructing reduced reaction mechanisms with given independent relations, Combust. Sci. Technol. 57 (1-3) (1988) 89-94.

[19] J. C. Keck, D. Gillespie, Rate-controlled partial-equilibrium method for treating reacting gas-mixtures, Combust. Flame 17 (1971) 237.

[20] U. Maas, S. B. Pope, Simplifying chemical kinetics: Intrinsic low-dimensional manifolds in composition space, Combust. Flame 88 (1992) 239-264.

[21] V. Bykov, U. Maas, The extension of the ILDM concept to reaction-diffusion manifolds, Combust. Theory Modelling 11 (2007) 839-862.

[22] Z. Ren, S. B. Pope, A. Vladimirsky, J. M. Guckenheimer, The invariant constrained equilibrium preimage curve method for the dimension reduction of chemical kinetics, J. Chem. Phys. 124 (2006) 114111.

[23] S. B. Pope, Computationally efficient implementation of combustion chemistry using in situ adaptive tabulation, Combust. Theory Modelling 1 (1997) 41-63.

[24] L. Lu, S. R. Lantz, Z. Ren, S. B. Pope, Computationally efficient implementation of combustion chemistry in parallel PDF calculations, J. Comput. Phys. 228 (2009) 54905525 .

[25] S. R. Tonse, N. W. Moriarty, N. J. Brown, F. M., PRISM: Piecewise reusable implementation of solution mapping. An economical strategy for chemical kinetics, Israel J. Chem. 39 (1999) 97-106.

[26] D. A. Schwer, P. Lu, W. H. Green, An adaptive chemistry approach to modeling complex kinetics in reacting flows, Combust. Flame 133 (4) (2003) $451-465$. 
[27] L. Liang, J. G. Stevens, J. T. Farrell, A dynamic adaptive chemistry scheme for reactive flow computations, Proc. Combust. Inst. 32 (1) (2009) 527-534.

[28] F. Contino, H. Jeanmart, T. Lucchini, G. D'Errico, Coupling of in situ adaptive tabulation and dynamic adaptive chemistry: An effective method for solving combustion in engine simulations, Proc. Combust. Inst. 33 (2) (2011) 3057-3064.

[29] L. Tosatto, B. A. V. Bennett, M. D. Smooke, A transport-flux-based directed relation graph method for the spatially inhomogeneous instantaneous reduction of chemical kinetic mechanisms, Combust. Flame 158 (5) (2011) 820-835.

[30] T. Løvas, S. Navarro-Martinez, S. Rigopoulos, On adaptively reduced chemistry in large eddy simulations, Proc. Combust. Inst. 33 (1) (2011) 1339-1346.

[31] B. J. Debusschere, Y. M. Marzouk, H. N. Najm, B. Rhoads, D. A. Goussis, M. Valorani, Computational singular perturbation with non-parametric tabulation of slow manifolds for time integration of stiff chemical kinetics, Combust. Theory Modelling 16 (1) (2012) $173-198$.

[32] M. Singer, W. Green, Using adaptive proper orthogonal decomposition to solve the reaction diffusion equation, Appl. Numer. Math 59 (2009) 272-279.

[33] I. Banerjee, M. Ierapetritou, An adaptive reduction scheme to model reactive flow, Combust. Flame 144 (3) (2006) 619-633.

[34] H. Yang, Z. Ren, T. Lu, G. M. Goldin, Dynamic adaptive chemistry for turbulent flame simulations, Combust. Theory Modelling 17 (1) (2013) 167-183.

[35] Z. Ren, C. Xu, T. Lu, M. A. Singer, Dynamic adaptive chemistry with operator splitting schemes for reactive flow simulations, J. Comput. Phys. 263 (2014) 19-36.

[36] A. Viggiano, V. Magi, Dynamic adaptive chemistry applied to homogeneous and partially stratified charge CI ethanol engines, Appl. Energ. 113 (2014) 848-863.

[37] X. Gou, Z. Chen, W. Sun, Y. Ju, A dynamic adaptive chemistry scheme with error control for combustion modeling with a large detailed mechanism, Combust. Flame 160 (2) (2013) $225-231$.

[38] Z. Ren, Y. Liu, T. Lu, L. Lu, O. O. Oluwole, G. M. Goldin, The use of dynamic adaptive chemistry and tabulation in reactive flow simulations, Combust. Flame 161 (1) (2014) $127-137$.

[39] K. He, I. P. Androulakis, M. G. Ierapetritou, Numerical investigation of homogeneous charge compression ignition (HCCI) combustion with detailed chemical kinetics using on-the-fly reduction, Energy Fuels 25 (8) (2011) 3369-3376. 
[40] N. J. Curtis, K. E. Niemeyer, C. J. Sung, An automated target species selection method for dynamic adaptive chemistry simulations, Combust. Flame 162 (4) (2015) 1358 1374.

[41] H. Wang, S. B. Pope, Large eddy simulation/probability density function modeling of a turbulent jet flame, Proc. Combust. Inst. 33 (1) (2011) 1319 - 1330.

[42] Y. Yang, H. Wang, S. B. Pope, J. H. Chen, Large-eddy simulation/probability density function modeling of a non-premixed $\mathrm{CO} / \mathrm{H} 2$ temporally evolving jet flame, Proc. Combust. Inst. 34 (1) (2013) $1241-1249$.

[43] V. Hiremath, S. R. Lantz, H. Wang, S. B. Pope, Computationally-efficient and scalable parallel implementation of chemistry in simulations of turbulent combustion, Combust. Flame 159 (2012) 3096-3109.

[44] K. E. Niemeyer, C.-J. Sung, On the importance of graph search algorithms for DRGEPbased mechanism reduction methods, Combust. Flame 158 (8) (2011) 1439-1443.

[45] Y. Yang, S. B. Pope, J. Chen, Empirical low-dimensional manifolds in composition space, Combust. Flame 160 (10) (2013) $1967-1980$.

[46] H. Curran, T. Jayaweera, W. Pitz, A detailed modeling study of propane oxidation, Western States Section of the Combustion Institute 2004.

[47] S. B. Pope, http://tcg.mae.cornell.edu/ell_lib, ELL_LIB: A Fortran library for ellipsoids. 